\title{
Auto- and cross-power spectral analysis of dual trap optical tweezer experiments using Bayesian inference
}

\author{
Yann von Hansen, ${ }^{1,2, a)}$ Alexander Mehlich, ${ }^{2}$ Benjamin Pelz, ${ }^{2}$ Matthias Rief, ${ }^{2}$ \\ and Roland R. Netz ${ }^{1,2}$ \\ ${ }^{1}$ Department of Physics, Freie Universität Berlin, 14195 Berlin, Germany \\ ${ }^{2}$ Physics Department, Technische Universität München, 85748 Garching, Germany
}

(Received 13 April 2012; accepted 22 August 2012; published online 28 September 2012)

\begin{abstract}
The thermal fluctuations of micron-sized beads in dual trap optical tweezer experiments contain complete dynamic information about the viscoelastic properties of the embedding medium and-if present-macromolecular constructs connecting the two beads. To quantitatively interpret the spectral properties of the measured signals, a detailed understanding of the instrumental characteristics is required. To this end, we present a theoretical description of the signal processing in a typical dual trap optical tweezer experiment accounting for polarization crosstalk and instrumental noise and discuss the effect of finite statistics. To infer the unknown parameters from experimental data, a maximum likelihood method based on the statistical properties of the stochastic signals is derived. In a first step, the method can be used for calibration purposes: We propose a scheme involving three consecutive measurements (both traps empty, first one occupied and second empty, and vice versa), by which all instrumental and physical parameters of the setup are determined. We test our approach for a simple model system, namely a pair of unconnected, but hydrodynamically interacting spheres. The comparison to theoretical predictions based on instantaneous as well as retarded hydrodynamics emphasizes the importance of hydrodynamic retardation effects due to vorticity diffusion in the fluid. For more complex experimental scenarios, where macromolecular constructs are tethered between the two beads, the same maximum likelihood method in conjunction with dynamic deconvolution theory will in a second step allow one to determine the viscoelastic properties of the tethered element connecting the two beads. () 2012 American Institute of Physics. [http://dx.doi.org/10.1063/1.4753917]
\end{abstract}

\section{INTRODUCTION}

Recent years have seen an ever increasing use of optical tweezers as sensitive tools for the manipulation and observation of single molecules, ${ }^{1}$ including a number of studies focusing on the mechanical properties of nucleic acids and proteins. ${ }^{2,3}$ As a result of technological development, spatial resolution with subnanometer precision and sampling rates reaching the $\mathrm{MHz}$ range ${ }^{4}$ are by now possible and allow unprecedented insights into molecular structure and dynamics. Similarly to micro-rheological studies, ${ }^{5}$ dual trap optical tweezers exhibit a number of advantages, including a higher "signal-to-noise" ratio and a minimized influence of instrumental $\mathrm{drift}^{6}$ and therefore are preferred to single trap setups, when high precision is a concern.

Typical extension traces recorded in equilibrium singlemolecule experiments display fluctuating trajectories with discrete jumps of the extension trace baseline: standard analysis consists of computing the moving average of the time series to identify distinct molecular conformations, their average extension, equilibrium probabilities and inter-state kinetics. ${ }^{3}$ The thermal fluctuations of the tethered beadsoften referred to as "noise"- are thus generally disregarded, although their spectral properties are a signature of the viscoelastic properties of the experimental construct and therefore interesting by themselves. In fact, based on coarse

a)E-mail: yann.von.hansen@fu-berlin.de. grained simulations of such a single-molecule experiment, the contributions of the experimental handles, of hydrodynamic interactions, and - most importantly — of the molecule under study to the measured fluctuations of the beads in their respective traps, could be disentangled. ${ }^{7}$

Within this paper, we provide a framework for the quantitative spectral analysis of data from actual dual trap optical tweezer experiments. As will be shown, this involves a detailed understanding of instrumental characteristics as well as a controlled way of inferring undetermined parameters from experimental data.

The manuscript is organized as follows: We start with a description of our experimental setup in Sec. II. The fluctuation dissipation theorem (FDT) relating thermal equilibrium fluctuations and linear force response of mechanical objects is reviewed in the general context of optical tweezer experiments in Sec. III A, while the more specific case of two unconnected beads is treated in Sec. III B. Low Reynolds number hydrodynamics and the resulting frequency dependence of self- and cross-mobilities are covered in Secs. IV A and IV B, respectively. We model the signal processing in a dual trap optical tweezer setup including polarization crosstalk, (parasitic) filtering, and instrumental noise and discuss the influence of the finite sampling rate on self- and cross power spectral densities (PSDs) in Secs. V A and V B. The statistical properties of auto- and cross-periodograms ${ }^{8}$ of the experimental time series in Sec. VI A form the basis for the maximum likelihood method presented in Sec. VI B, which by Bayesian inference 
allows to determine optimal values for the model parameters. Judging whether the theoretical model used is indeed adequate may be done by quantifying the deviations between fit and experimental data as explained in Sec. VI C. The overall procedure for the spectral analysis is summarized in Sec. VI D. As an application of the maximum likelihood method, we suggest a calibration scheme for dual trap optical tweezers based on three measurements in Sec. VII; it can be viewed as a generalization of a calibration technique, which for single trap setups is by now standard. ${ }^{9-13}$ We consider a pair of unconnected, but hydrodynamically interacting beads trapped in their respective laser foci in Sec. VIII and explicitly demonstrate the influence of instrumental effects and of finite statistics on the auto- and cross-periodograms of the measured signals. For a carefully calibrated setup, PSDs estimated from experimental data agree with the theoretical predictions taking into account the frequency dependence of hydrodynamic interactions (HI) arising from the finite time for vortex diffusion in the fluid. ${ }^{14}$ Our main findings are summarized in Sec. IX, while more technical aspects are found in the appendices: Details concerning the notation are provided in Appendix A, various filter effects are discussed in Appendix B, statistical properties of auto- and crossperiodograms are explicitly calculated in Appendix $\mathrm{C}$, basic characteristics of the discrete Fourier transform (DFT) are reviewed in Appendix D, and the properties of normal distributed random variables on logarithmic plots are discussed in Appendix E.

For more complex experimental situations, where a pair of trapped beads are, e.g., tethered via double stranded DNA handles to a protein, we suggest a two-step procedure to reliably determine the viscoelastic properties of the molecular tether: (i) a calibration run as described in Sec. VII without the tethering macromolecular construct to fix the instrumental parameters, and (ii) a production run with the tether construct to determine the tether parameters. In both steps, the same maximum likelihood method (Sec. VI B) can be used, the only difference lying in the thermal PSDs (Sec. III A) and in the set of parameters to be determined.

\section{DUAL TRAP OPTICAL TWEEZERS}

The experimental setup used for trapping and tracking micron-sized beads is a custom-built high-resolution dual trap optical tweezer with back-focal plane detection (see Fig. 1(a)) similar to setups described elsewhere. ${ }^{6,12,15}$ The light of a $4 \mathrm{~W}$ linear polarized diode pumped Nd:YVO4 solid state continuous wave laser system emitting at $1064 \mathrm{~nm}$ (Spectra-Physics, California, USA) passes a Faraday isolator (Electro-Optics Technology, Inc., Michigan, USA) before getting expanded and collimated by the first telescope. A first computer controlled and motorized $\lambda / 2$-plate followed by a polarizing beam splitter (PBS) cube (order number PTW 2.10 or PTW 2.20, Bernhard Halle, Germany) controls the overall laser power passing into the successive optical parts. A second $\lambda / 2$-plate sets the relative laser power in the two orthogonally polarized trapping beams separated by a second PBS. One of the beams is reflected by a mirror mounted on a two axis piezoelectric tip/tilt actuator (Mad City Labs, Wisconsin, USA) provid- ing lateral displacement of the corresponding trap in the specimen plane. The other beam is frequency shifted using an acousto-optical modulator (Gooch \& Housego, Great Britain) to reduce interference artifacts. ${ }^{16}$ After recombination of the two trapping beams with a third PBS a second telescope expands the beams to overfill the back aperture of the focusing objective (63x/1.20 W Corr, C-Apochromat, Zeiss, Germany). The objective focuses the beams to two diffraction-limited spots in the sample chamber. The use of a water-immersion objective permits trapping deep inside the sample chamber: the distance from the cover slip is set to $20 \mu \mathrm{m}$ and therewith is much larger than typical inter-bead distances; the influence of the cover-slip surface on the hydrodynamics is therefore neglected in Sec. IV. After passing the sample chamber placed on a piezoelectric table (Physik Instrumente, Germany), the forward scattered light of the trapped beads is collimated with a second identical objective. The beams are split by polarization and imaged onto two position sensing devices (DL100-7PCBA3, Pacific Silicon Sensor Inc., California, USA). Due to depolarization in the optical path and the intrinsic non-perfect separation and conservation of polarization upon transmission through and reflection by the PBSs, each of the detector signals to a certain degree also reflects the motion of the bead in the "wrong" trap; this effect, known as polarization crosstalk, ${ }^{17}$ is explicitly considered in the signal processing described in Sec. V A. All components are mounted on a vibration isolated optical table (M-ST-4612, Newport Corporation, Irvine, USA). Optical paths are enclosed to minimize the effect of air fluctuations. Custom-built electronics are used for processing the analog position signals including an individual offset correction, normalization, and amplification for each channel..$^{9,18}$ Before recording, all signals are filtered with an eighth order Butterworth filter with $3 \mathrm{~dB}$-frequency set at $200 \mathrm{kHz}$ and further amplified (Model 3384 Filter, Kron-Hite, Massachusetts, USA). For real-time steering and data acquisition a custom-written LABVIEW program runs on a field-programmable gate array (FPGA)-board (NI PCI-7833R 3M, National Instruments, Texas, USA). Data is recorded with a sampling rate of $100 \mathrm{kHz}$. Measurements are performed at a temperature of $22.6 \pm 0.3{ }^{\circ} \mathrm{C}$ using silica beads with $1.0 \mu \mathrm{m}$ in diameter (Bangs Laboratories, Indiana, USA) diluted in phosphate buffered saline (P4417, Sigma Aldrich, St. Louis, USA). The sample chamber consists of two cover slips separated by stripes of Nescofilm (Bando Chemical Industries Ltd., Japan) forming a channel, which is sealed with vacuum grease after filling. The dimensions of the sample chamber are about $9 \times 18 \times 0.1 \mathrm{~mm}^{3}$.

\section{FORCE RESPONSE AND THERMAL MOTION}

\section{A. General formulation}

In a dual trap optical tweezer experiment, the thermal motion of the two beads in their respective traps reflects the viscoelastic properties of the experimental construct. For simplicity, we restrict the following discussion to the case, in which all spatial components decouple; the necessary conditions for this to happen are detailed in Sec. III B. The thermal cross-correlation function of the bead coordinates $r_{i}$ and $r_{j}$ is 
defined as

$$
\begin{aligned}
S_{i j}^{\text {th }}(t) & \equiv\left\langle r_{i}(0) r_{j}(t)\right\rangle=\left\langle r_{i}\left(t^{\prime}\right) r_{j}\left(t^{\prime}+t\right)\right\rangle_{t^{\prime}} \\
& \equiv \lim _{T \rightarrow \infty} \frac{1}{T} \int_{-T / 2}^{T / 2} \mathrm{~d} t^{\prime} r_{i}\left(t^{\prime}\right) r_{j}\left(t^{\prime}+t\right), \quad i, j=1,2,
\end{aligned}
$$

where the equivalence of equilibrium average $\langle\ldots\rangle$ and temporal average $\langle\ldots\rangle_{t^{\prime}}$ holds in ergodic systems and where the measurement time is denoted by $T$. Correlation functions in thermal equilibrium are of interest because they reflect the linear force response properties of the system; in fact, the FDT ${ }^{19}$ states

$$
J_{i j}(t)= \begin{cases}-\left(k_{\mathrm{B}} T\right)^{-1} \mathrm{~d} S_{i j}^{\mathrm{th}}(t) / \mathrm{d} t & \text { for } t \geq 0, \\ 0 & \text { for } t<0,\end{cases}
$$

where $k_{\mathrm{B}} T$ denotes the thermal energy and $J_{i j}$ the corresponding response function. In the past, the interplay of (instantaneous) $\mathrm{HI}$ and the harmonic laser trap potential ${ }^{20}$ as well as the relaxation dynamics of $\mathrm{DNA}^{21}$ were measured resolving auto- and cross-correlation functions of the bead's positions.

Here, working in frequency space turns out to be more convenient for the following reasons: (i) Retardation/memory effects in the (integro-differential) equations of motions cause difficulties when calculating temporal correlation functions, while they are easily taken into account in frequency space as shown in Sec. III B, (ii) relevant instrumental effects such as crosstalk, signal filtering, and instrumental noise can be accounted for as detailed in Sec. V, and (iii) the maximum likelihood method presented in Sec. VI B makes it straightforward to quantitatively evaluate experimental estimates for autoand cross-PSDs, defined as the Fourier transformations (cf. Appendix A) of the corresponding thermal correlation functions

$$
S_{i j}^{\mathrm{th}}(\omega) \equiv \int_{-\infty}^{\infty} \mathrm{d} t S_{i j}^{\mathrm{th}}(t) \mathrm{e}^{\mathrm{i} \omega t}, \quad i, j=1,2 .
$$

Note that if time reversal symmetry holds, temporal correlation functions $S_{i j}^{\text {th }}(t)$ as well as their Fourier transformations, the PSDs $S_{i j}^{\text {th }}(\omega)$ are real and even functions. According to the cross-correlation theorem, PSDs and the Fourier components of the stochastic motion are related via

$$
S_{i j}^{\mathrm{th}}(\omega) 2 \pi \delta\left(\omega-\omega^{\prime}\right)=\left\langle r_{i}(\omega) r_{j}^{\star}\left(\omega^{\prime}\right)\right\rangle,
$$

and PSDs can thus directly be estimated experimentally by calculating averaged auto- and cross-periodograms from the stochastic trajectories as detailed in Sec. VI A.

For simple systems as the one in Sec. III B, thermal PSDs can be obtained easily from the stochastic equations of motion; for more complex constructs, it may be easier to calculate linear response functions $J_{i j}$ relating average oscillation and external driving force amplitudes on the linear level

$$
\left\langle r_{i}(\omega)\right\rangle=\sum_{j=1}^{2} J_{i j}(\omega) f_{j}^{\mathrm{ext}}(\omega), \quad i=1,2 .
$$

Overall, the experimental object of study-two micron-sized beads in optical traps potentially connected via a molecular tether and embedded in a fluid-is completely characterized by two self-response functions $J_{11}$ and $J_{22}$ relating forces and displacements on the same bead and, in the absence of time reversal breaking, one cross-response function $J_{12}=J_{21}$ relating force and displacement amplitudes of different beads. Dynamic (de-) convolution theory ${ }^{7}$ provides a framework for iteratively calculating these three response functions from the mechanical response characteristics of the individual components, i.e., the beads in the optical traps, the elements of the molecular tether and HI between the various components. The corresponding thermal PSDs are then obtained using the FDT in frequency space

$$
S_{i j}^{\mathrm{th}}(\omega)=\frac{2 k_{\mathrm{B}} T}{\omega} \operatorname{Im}\left[J_{i j}(\omega)\right],
$$

where $\operatorname{Im}[\ldots]$ denotes the imaginary part. Because of causality $\left(J_{i j}(t)=0\right.$ for times $\left.t<0\right)$, real and imaginary parts of the response functions in frequency space are related by KramersKronig relations. ${ }^{19}$ Recording the equilibrium fluctuations of the system and estimating the PSDs $S_{i j}^{\text {th }}$ is therefore sufficient for completely characterizing the system's force response via Eq. (6).

\section{B. Hydrodynamically interacting beads}

The stochastic, thermal motion of two unconnected beads in the trapping potentials of two laser foci is described by a set of differential equations, which in the frequency domain reads

$$
\begin{aligned}
-\mathrm{i} \omega\left(\begin{array}{l}
\boldsymbol{r}_{1}(\omega) \\
\boldsymbol{r}_{2}(\omega)
\end{array}\right)= & \left(\begin{array}{ll}
\overleftrightarrow{\boldsymbol{\mu}}_{11}(\omega) & \overleftrightarrow{\boldsymbol{\mu}}_{12}(\omega) \\
\overleftrightarrow{\boldsymbol{\mu}}_{21}(\omega) & \overleftrightarrow{\boldsymbol{\mu}}_{22}(\omega)
\end{array}\right) \\
& \cdot\left(\begin{array}{c}
\boldsymbol{f}_{1}^{\mathrm{trap}}(\omega)+\boldsymbol{f}_{1}^{\mathrm{ext}}(\omega)+m_{1} \omega^{2} \boldsymbol{r}_{1}(\omega) \\
\boldsymbol{f}_{2}^{\mathrm{trap}}(\omega)+\boldsymbol{f}_{2}^{\mathrm{ext}}(\omega)+m_{2} \omega^{2} \boldsymbol{r}_{2}(\omega)
\end{array}\right) \\
& +\left(\begin{array}{c}
\boldsymbol{v}_{1}^{\mathrm{st}}(\omega) \\
\boldsymbol{v}_{2}^{\mathrm{st}}(\omega)
\end{array}\right) .
\end{aligned}
$$

Here, the $3 \times 3$ mobility tensors $\overleftrightarrow{\boldsymbol{\mu}}_{i j}$ relate the forces on bead $j$, i.e., forces due to the trapping potential $f_{j}^{\text {trap }}$, external forces $\boldsymbol{f}_{j}^{\text {ext }}$, and inertial ones $\left(m_{1}\right.$ and $m_{2}$ denoting the beads' masses), to the velocity $-\mathrm{i} \omega \boldsymbol{r}_{i}$ of bead $i$; the stochastic contribution to the motion of bead $i$ is denoted by $\boldsymbol{v}_{i}^{\text {st }}$.

The motion along the individual spatial coordinates in Eq. (7) decouples if the main axis of the elliptic laser foci coincide with the cartesian coordinates ${ }^{9}$ and if the mobility tensors are diagonal. While the first condition can be achieved via careful alignment of the instrument, ${ }^{22}$ the second requirement is satisfied in an unbounded fluid if the axis connecting the trap centers is parallel to one of the coordinate axes (cf. Sec. IV B). To simplify the notation, we restrict ourselves to two identical beads ( $m \equiv m_{1}=m_{2}$ ) in the following; we distinguish motion along the optical axis $(o)$ as well as along $(\|)$ and perpendicular $(\perp)$ to the inter-trap axis as shown in Fig. 1(b).

The thermal motion of the two beads held in harmonic traps along the spatial coordinate $\gamma$ is then described by the 


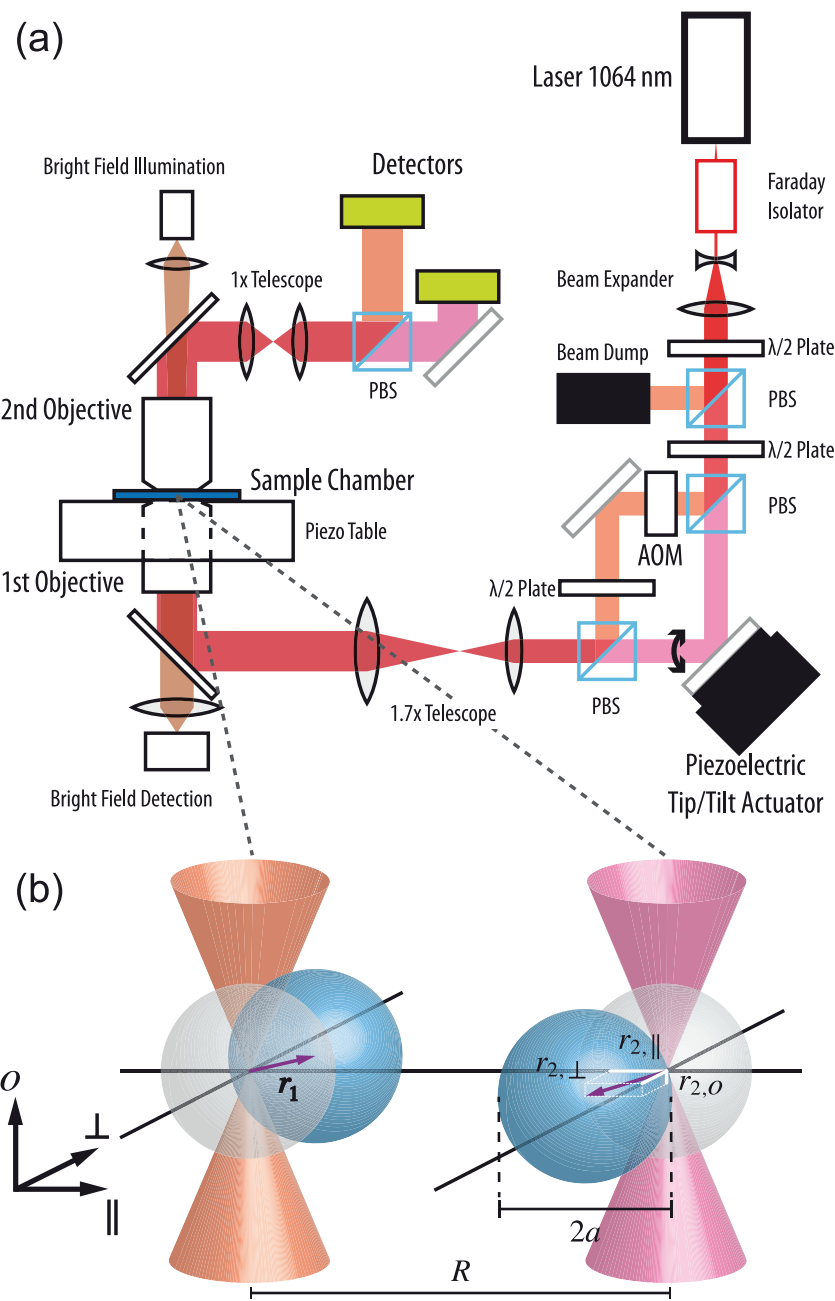

FIG. 1. (a) Schematic of the experimental setup described in Sec. II. (b) Zoom-in into the sample chamber: The notation for the coordinates of the beads (blue) relative to the trap centers (gray) is used throughout the manuscript.

simpler set of equations of motion

$$
\begin{aligned}
-\mathrm{i} \omega\left(\begin{array}{l}
r_{1, \gamma}(\omega) \\
r_{2, \gamma}(\omega)
\end{array}\right)= & \left(\begin{array}{cc}
\mu_{\text {self }}(\omega) & \mu_{\text {cross }}^{\gamma}(\omega) \\
\mu_{\text {cross }}^{\gamma}(\omega) & \mu_{\text {self }}(\omega)
\end{array}\right) \\
& \cdot\left(\begin{array}{c}
\left(-\kappa_{1, \gamma}+m \omega^{2}\right) r_{1, \gamma}(\omega)+f_{1, \gamma}^{\text {ext }}(\omega) \\
\left(-\kappa_{2, \gamma}+m \omega^{2}\right) r_{2, \gamma}(\omega)+f_{2, \gamma}^{\text {ext }}(\omega)
\end{array}\right) \\
& +\left(\begin{array}{c}
v_{1, \gamma}^{\text {st }}(\omega) \\
v_{2, \gamma}^{\text {st }}(\omega)
\end{array}\right), \quad \gamma=o, \|, \perp,
\end{aligned}
$$

where the trap strengths $\kappa_{i, \gamma}$ and, in particular, the crossmobilities $\mu_{\text {cross }}^{\gamma}$ in general depend on the spatial coordinate $\gamma$. The frequency dependence of self- and cross-mobilities is a consequence of the hydrodynamic flow field created around a moving sphere discussed in Sec. IV. The stochastic velocity contributions $v_{i, \gamma}^{\text {st }}$ in Eq. (8) are due to thermal kicks of solvent molecules and are normal distributed random variables with vanishing mean

$$
\left\langle v_{i, \gamma}^{\mathrm{st}}\right\rangle=0, \quad i=1,2, \quad \gamma=o, \|, \perp,
$$

and correlations

$$
\begin{aligned}
& \left\langle v_{i, \gamma}^{\mathrm{st}}(\omega) v_{j, \gamma}^{\mathrm{st}}{ }^{\star}\left(\omega^{\prime}\right)\right\rangle=4 \pi k_{\mathrm{B}} T \operatorname{Re}\left[\delta_{i j} \mu_{\text {self }}(\omega)\right. \\
& \left.+\left(1-\delta_{i j}\right) \mu_{\text {cross }}^{\gamma}(\omega)\right] \delta\left(\omega-\omega^{\prime}\right),
\end{aligned}
$$

where $(\ldots)^{\star}$ denotes the complex conjugate and $\operatorname{Re}[\ldots]$ the real part. Equation (8) being linear, solving for $r_{i, \gamma}$ is straightforward; for each of the spatial directions $\gamma$, thermal PSDs $S_{i j}^{\gamma}$,th can then be either calculated directly using Eq. (4) or by identifying response functions $J_{i j}^{\gamma}$ defined via Eq. (5) and using the FDT (Eq. (6)).

Though being in general characterized by a weaker trapping strength, the coordinate $o$ is equivalent to the coordinate $\perp$ from a hydrodynamic point of view due to symmetry, cf. Sec. IV. Since bead displacements along $\perp$ are easier to access experimentally than the ones along $o,{ }^{23}$ we restrict the following discussion to the coordinates $\|$ and $\perp$.

Note that for tethered beads, motion along $o$ and $\perp$ will in general couple to the equations of motion for $r_{i, \|}$ even in a perfectly aligned setup. These non-linear contributions can however be suppressed using longer tethers and smaller bead sizes. ${ }^{6,7}$

\section{LOW REYNOLDS NUMBER HYDRODYNAMICS}

\section{A. Flow-field around an oscillating sphere}

The Reynolds number characterizing the relative importance of inertial compared to viscous forces in a hydrodynamic flow is typically small for the thermal motion of micron-sized objects in water: for a bead of radius $a$ moving with velocity $v$ through a fluid of kinematic viscosity $v$ $\equiv \eta / \rho$, with the fluid's shear viscosity being denoted by $\eta$ and its mass density by $\rho$, one has $R e \sim a v / v$. The equipartition theorem $k_{\mathrm{B}} T \sim m\left\langle v^{2}\right\rangle$, where the bead's mass is denoted by $m$, allows to estimate the Reynolds numbers for the thermal motion of a micron-sized bead in water, $R e \sim \sqrt{k_{\mathrm{B}} T / m} a / v$ $\sim 10^{-3} \ll 1$. Hydrodynamics are thus adequately described by the linearized Navier-Stokes (or transient Stokes) equation, which for an incompressible fluid reads

$$
\rho \partial \boldsymbol{u} / \partial t=\eta \Delta \boldsymbol{u}-\nabla p, \quad \nabla \cdot \boldsymbol{u}=0,
$$

where $\boldsymbol{u}$ denotes the velocity and $p$ the pressure field in the fluid. Taking the curl of Eq. (9) yields a diffusion equation for the vorticity $\boldsymbol{\Omega} \equiv \nabla \times \boldsymbol{u}$ : vortices due to forces or torques acting on objects in a viscous fluid thus diffusively propagate in space, the characteristic time scale for diffusion over a distance $R$ being $\tau_{R} \equiv R^{2} / \nu$. A prominent consequence of the finite time scale for vortex diffusion is the frequency dependence of the self-mobility $\mu_{\text {self }}$ (the inverse of the sphere's drag coefficient): For a no-slip sphere of radius $a$ in an unbounded and incompressible fluid, the exact result found by Stokes $^{24}$ reads

$$
\mu_{\text {self }}^{\text {Stokes }}(\omega)=\mu_{0} /\left(1+\alpha a+\alpha^{2} a^{2} / 9\right) .
$$

where $\alpha$ denotes the inverse penetration depth

$$
\alpha \equiv \sqrt{-\frac{\mathrm{i} \omega}{v}}=\frac{\sqrt{-\mathrm{i} \omega \tau_{a}}}{a}, \quad \operatorname{Re}[\alpha]>0,
$$

and $\tau_{a} \equiv a^{2} / \nu$ is the characteristic time scale for vortex diffusion on length scales comparable to the bead's radius $a$; the self-mobility thus deviates from the quasi-stationary self-mobility $\mu_{0} \equiv 1 /(6 \pi \eta a)$ for frequencies $\omega \gtrsim \tau_{a}^{-1}$. Though theoretically known for over a century, the frequency 

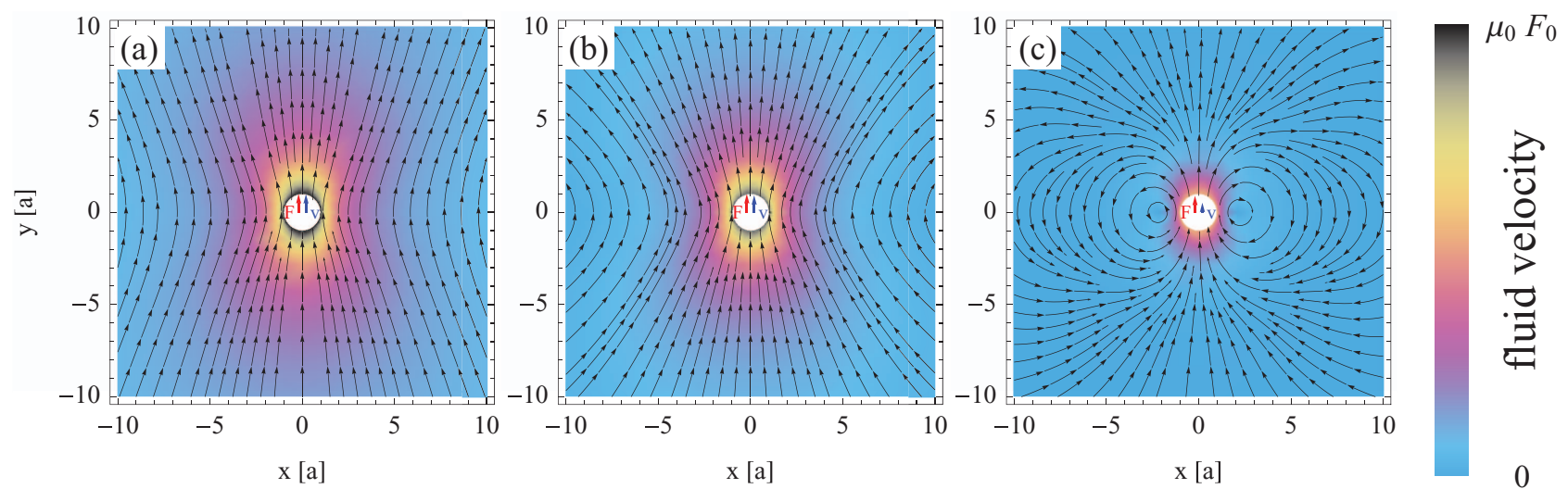

FIG. 2. Cross sections of the flow field around an oscillating no-slip sphere in an incompressible fluid for various driving frequencies: (a) $\omega=10^{-4} / \tau_{a}$, (b) $\omega$ $=10^{-2} / \tau_{a}$, (c) $\omega=1 / \tau_{a}$. Streamlines indicate the direction of the velocity field and the color coding its magnitude, where $F_{0}$ is the driving force amplitude and $\mu_{0}=1 /(6 \pi \eta a)$ the quasi-static sphere mobility. Spatial coordinates are given in units of the sphere radius $a$, frequencies in units of $\tau_{a}^{-1}=v / a^{2}$, the kinematic viscosity of the fluid being denoted by $v$. Amplitudes of the driving force acting on and the velocity of the sphere are shown as red and blue arrows within the spheres. The figures above show the configuration of the flow-field, when the sphere velocity is maximal; animations showing the time evolution of these flow fields are available online. ${ }^{27}$

dependence in Eq. (10)—responsible for power law tails in the velocity autocorrelation function and resonances in power spectral densities - is of ongoing interest. ${ }^{4,25}$

The velocity field around an oscillating sphere with noslip boundary condition on its surface is calculated employing a standard singularity ansatz involving the Green's function for a force Stokeslet. ${ }^{24,26}$ For a sphere in an incompressible fluid, cross sections of the velocity field for various values of $\omega \tau_{a}$ are shown in Fig. 2; animations visualizing the time evolution of these velocity fields are available online. ${ }^{27}$ The direction of the velocity field is denoted by streamlines (black lines with arrow heads), its amplitude by the color coding, which is the same in all snapshots and movies, emphasizing the attenuation of the velocity field at higher frequencies. At low frequencies the time dependence in Eq. (9) can be neglected: in the "creeping flow" limit, forces are instantaneously propagated through the fluid. The animations for values $\omega \lesssim 10^{-4} / \tau_{a}$ indeed show a flow-field that is almost immediately built up all over the displayed cross section; the flow-field in Fig. 2(a) is long-ranged decaying as $1 / R$ with corrections accounting for the finite bead size at small separations $R \sim a$. In contrast, retardation/memory effects become noticeable, when the time scale $\tau_{R}$ for vortex diffusion over a distance $R$ becomes comparable to the oscillation period: the flow direction changes and the velocity magnitude is attenuated as seen in Fig. 2(b). For further increasing frequency, vorticity effects not only appear at the edges of the displayed cross section, but also in the sphere's vicinity. For $\omega=1 / \tau_{a}$ (Fig. 2(c)), the subsequent creation of vortices and their diffusion towards the sides is best seen. For even larger frequencies $\left(\omega \tau_{a} \gtrsim 10\right)$ shown in the series of animations, ${ }^{27}$ the flow-field is completely changed: it is now dominated by the dipole contribution with magnitude decaying like $1 / R^{3}$ and with frequency as $1 / \omega$, while vorticity effects are restricted to the direct proximity of the sphere. The external force acting on and the velocity of the sphere are related by the frequency-dependent self-mobility given in Eq. (10). Their amplitudes are shown as red and blue arrows within the spheres in Fig. 2 and in the animations; $;^{27}$ the phase shift and the decrease of the sphere's self-mobility are clearly discernible in the regime $\omega \tau_{a} \gtrsim 1$.

Similarly to the sphere's self-mobility (Eq. (10)), the strength of HI, i.e., the entrainment of objects by the flow fields shown in Fig. 2, also depends on the driving frequency as discussed in Sec. IV B. For the spheres of radius $a=500 \mathrm{~nm}$ used in our experiment, the vorticity time scale in water is $\tau_{a} \approx 0.26 \mu \mathrm{s}$; in the snapshots (Fig. 2) and animations, ${ }^{27} \omega=10^{-2} / \tau_{a}$ thus corresponds to a driving frequency $f \approx 6 \mathrm{kHz}$.

\section{B. Frequency dependence of self- and cross-mobilities}

Calculating the HI between two spheres of radius $a$ at radial distance $R$ constitutes a challenging problem from a mathematical point of view, because the no-slip boundary conditions on the sphere's surfaces have to be fulfilled simultaneously. Since no closed-form solution is known, one has to rely on approximations of varying complexity and accuracy, which are discussed in the following. A series expansion of self- and cross-mobilities in powers of $a / R$ and $\alpha a$ by van Saarloos and Mazur ${ }^{28}$ (vSM) yields self-mobilities

$$
\mu_{\mathrm{self}}^{\mathrm{vSM}}(\omega)=\mu_{0}\left(1-\alpha a+\frac{8}{9} \alpha^{2} a^{2}-\frac{7}{9} \alpha^{3} a^{3}\right)
$$

and cross-mobilities

$$
\begin{aligned}
\frac{\mu_{\text {cross }}^{\gamma, \mathrm{vSM}}(\omega)}{\mu_{0}}= & \frac{3 a}{4 R} \mathrm{e}^{-\alpha R}\left(1+\frac{5}{9} \alpha^{2} a^{2}+\frac{1}{6} \alpha^{3} a^{3}\right)\left[1+\delta_{\gamma, \|}\right] \\
& -\left[\frac{1}{3}-\delta_{\gamma, \|}\right] \times\left(\frac{a^{3}}{R^{3}}+\frac{9 a}{2 \alpha^{2} R^{3}}\right. \\
& \left.-\frac{\left(5 \alpha^{2} a^{2}+9\right)\left(\alpha^{2} R^{2}+2 \alpha R+2\right) a}{4 \alpha^{2} R^{3}} \mathrm{e}^{-\alpha R}\right),
\end{aligned}
$$




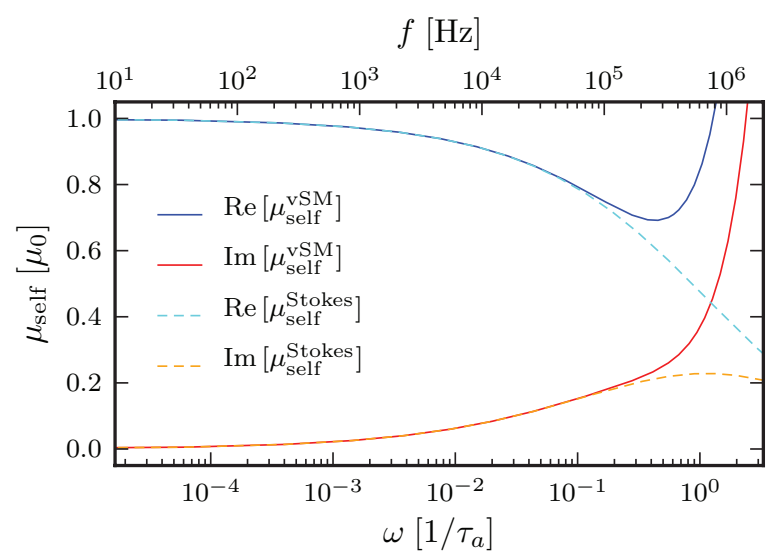

FIG. 3. Dependence of real and imaginary parts of the self-mobility of a sphere on the driving frequency $\omega$. In the low-frequency limit, the quasistationary self-mobility $\mu_{0} \equiv 1 /(6 \pi \eta a)$ of a sphere of radius $a$ in a fluid of shear viscosity $\eta$ is recovered. On the lower axis, the angular frequency $\omega$ is rescaled by $\tau_{a} \equiv a^{2} / \nu$, the upper frequency scale corresponds to the experimental case where $\tau_{a} \approx 0.26 \mu$ s (beads of radius $a=500 \mathrm{~nm}$ in water). The exact result by Stokes (dashed lines, Eq. (10)) and the approximate expressions (solid lines, Eq. (12)) nicely match over the entire range of frequencies resolved in our experiment $(f \lesssim 100 \mathrm{kHz})$.

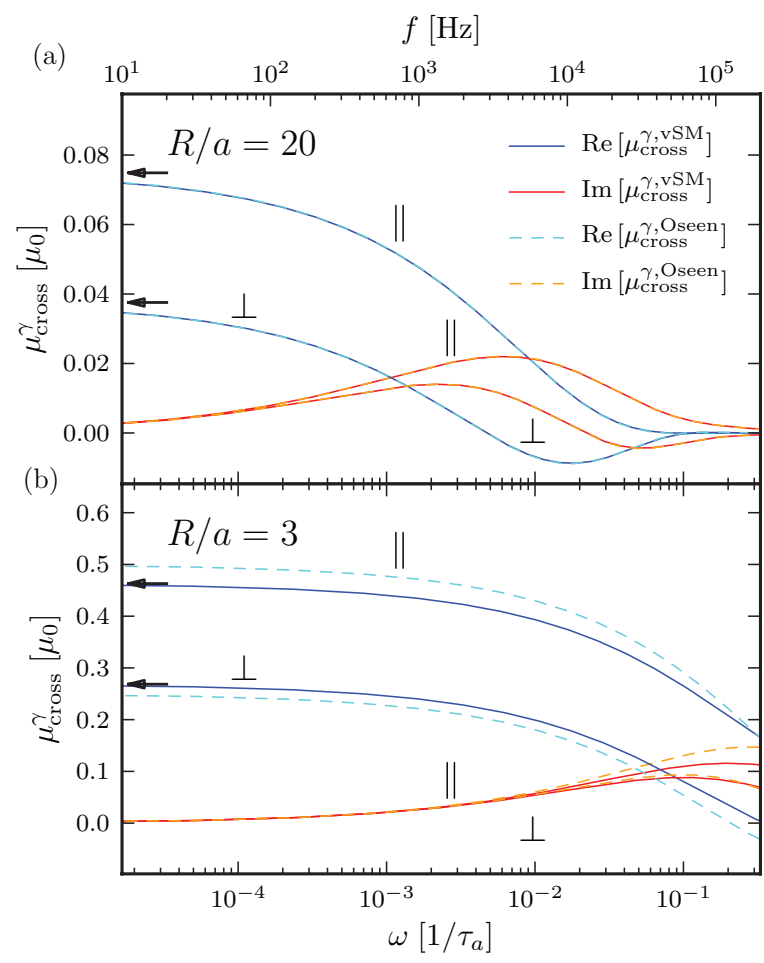

FIG. 4. Frequency dependence of cross-mobilities parallel $(\gamma=\|)$ and perpendicular $(\gamma=\perp)$ to the inter-bead axis for different ratios of inter-bead separation $R$ to bead radius $a$ : (a) $R / a=20$, (b) $R / a=3$. Cross-mobilities for spheres of finite radius (solid lines, Eq. (13)) are compared to the Oseen result for point-like particles (dashed lines, Eq. (15)) and to quasi-static HI (horizontal arrows, Eq. (14)). Cross-mobilities are given in units of the quasistatic Stokes self-mobility $\mu_{0} \equiv 1 /(6 \pi \eta a)$ for a sphere of radius $a$ in a fluid of viscosity $\eta$; the angular frequency is rescaled by the characteristic time scale $\tau_{a}$ for vortex diffusion over the length scale of the bead's radius $a$, the upper frequency scale corresponds to experimental conditions ( $\left.\tau_{a} \approx 0.26 \mu \mathrm{s}\right)$. where $\delta_{\|,\|}=1$ and $\delta_{\perp, \|}=0$. A comparison of the approximate expression for the spheres' self-mobility (Eq. (12)) and the exact result by Stokes (Eq. (10)) is shown in Fig. 3: both nicely match over the entire frequency range accessible in our experiment $(f \lesssim 100 \mathrm{kHz})$.

The frequency dependence of the cross-mobilities parallel and perpendicular to the inter-bead axis are shown for different ratios of inter-bead separation $R$ to bead radius $a$ as solid lines in Fig. 4. In the low-frequency limit $\omega \tau_{R} \rightarrow 0$, the cross-mobilities in Eq. (13) reduce to the principal components of the Rotne-Prager (RP) tensor ${ }^{29}$ commonly employed in Brownian hydrodynamics simulations ${ }^{30}$

$$
\frac{\mu_{\text {cross }}^{\gamma, \mathrm{RP}}}{\mu_{0}}=\frac{3 a}{4 R}\left[1+\delta_{\gamma, \|}\right]+\frac{3 a^{3}}{2 R^{3}}\left[\frac{1}{3}-\delta_{\gamma, \|}\right],
$$

with dominant $1 / R$-scaling and corrections accounting for the finite bead size $a$. These quasi-stationary cross-mobilities are indicated by horizontal arrows in Fig. 4 .

The finite time scale $\tau_{R}$ for the vorticity to diffuse over length scales comparable to the bead separation $R$ is reflected in the frequency dependence of real and imaginary parts of the cross-mobilities as can be seen in Fig. 4; corrections to the quasi-static limit denoted by horizontal arrows, set in at frequencies $\omega \sim 1 / \tau_{R}$ and thus become apparent at smaller frequencies for the case of large separations $R / a=20$ as compared to $R / a=3$. In contrast, corrections accounting for the finite size of the beads are important at small separations $(R / a=3)$ and become irrelevant at larger ones $(R / a=20)$ : as expected, in the limit $a / R \rightarrow 0$ the expressions for the cross-mobilities (Eq. (13)) reduce to the Oseen result for point-forces ${ }^{31,32}$

$$
\begin{aligned}
\frac{\mu_{\text {cross }}^{\gamma, \text { Oseen }}}{\mu_{0}}= & \frac{3 a\left(1-e^{-\alpha R}(\alpha R+1)\right)}{\alpha^{2} R^{3}} \delta_{\gamma, \|} \\
& +\frac{3 a}{2} \frac{e^{-\alpha R}\left(\alpha^{2} R^{2}+\alpha R+1\right)-1}{\alpha^{2} R^{3}}\left[1-\delta_{\gamma, \|}\right],
\end{aligned}
$$

shown as dashed lines in Fig. 4. In the frequency and distance range, in which $|\alpha| R \gg 1$, but still $|\alpha| a \ll 1$, Eq. (13) reduces to $^{28}$

$$
\frac{\mu_{\text {cross }}^{\gamma}(\omega)}{\mu_{0}} \approx-\left(\frac{9}{2} \frac{a}{\alpha^{2} R^{3}}+\frac{a^{3}}{R^{3}}\right)\left[\frac{1}{3}-\delta_{\gamma, \|}\right],
$$

revealing the existence of a second dynamic regime, in which $\mathrm{HI}$ are considerably weakened and decay as $1 / R^{3}$. Note that since $|\alpha| a \ll 1$, in the above expression the first term $\propto \omega^{-1}$ dominates over the second one accounting for the finite size of the beads.

As discussed in the last paragraphs, the strength of HI depends on the relative distance and orientation of the two beads, making the equations of motion (Eqs. (7) and (8)) non-linear. The fact that the bead's positional fluctuations-in our setup on the order of $\sqrt{k_{\mathrm{B}} T / \kappa} \approx 5 \mathrm{~nm}$-are relatively small compared to the average inter-bead separation $R$ $\sim 1.5-10 \mu \mathrm{m}$ justifies the pre-averaging ${ }^{33}$ of the equations of motion, i.e., replacing the actual cross-mobilities by the (constant) cross-mobilities for the average bead configuration. 
Previous experimental studies have mostly focused on the quasi-stationary limit (Eq. (14)): The interplay of instantaneous HI and the finite relaxation times in the harmonic trapping potentials of optical tweezers are responsible for time-delayed anti-correlations in the beads' positions. ${ }^{20}$ Similarly, translation-rotation coupling, though decaying as $1 / R^{2}$ and therefore weaker, is equally observable combining optical tweezers with polarization microscopy. ${ }^{34}$ The frequency dependence of $\mathrm{HI}$ has been addressed in a series of experiments ${ }^{14}$ based on the point-particle limit derived by Oseen (Eq. (15)), thereby neglecting corrections for the finite size of the beads.

\section{SIGNAL PROCESSING IN A DUAL TRAP OPTICAL TWEEZER EXPERIMENT}

The electric signals recorded in a dual trap optical tweezer setup as described in Sec. II in general differ from the actual trajectories of the two beads due to various instrumental effects ${ }^{9,17,35}$ including polarization crosstalk, filtering of the position sensing device and amplification electronics as well as instrumental noise; the consequences of these instrumental effects on the PSDs are discussed in Sec. V A. Experimentally, PSDs are estimated based on discretely sampled and finite trajectories: resulting effects are addressed in Sec. V B.

Notation: The following discussion equally applies to the signals corresponding to both spatial coordinates $(\gamma=\|, \perp)$; however, to avoid an overloaded notation, indices, and superscripts $\gamma$ are omitted.

\section{A. Crosstalk, (parasitic) filtering, and noise}

The use of a single laser for both optical traps induces polarization crosstalk between the channels, ${ }^{17}$ meaning that changes in the positions $r_{1}$ and $r_{2}$ of both beads contribute to each of the detected signals

$$
\tilde{z}_{i}(t) \equiv \int_{-\infty}^{\infty} \mathrm{d} t^{\prime} f_{i}\left(t-t^{\prime}\right) \sum_{j=1}^{2} \epsilon_{i j} r_{j}\left(t^{\prime}\right) \quad i=1,2,
$$

with amplitudes $\epsilon_{11} \gg \epsilon_{12}$ and $\epsilon_{22} \gg \epsilon_{21}$ and where $f_{i}$ denotes the filter kernel in channel $i$. Filtering can be due to the position sensing device detecting the centroid of the laser spot intensity, ${ }^{11,35}$ the amplification electronics or other factors, which are discussed in detail in Appendix B. In addition, the recorded signals are affected by stationary additive and independent instrumental noise

$$
z_{i}(t)=\tilde{z}_{i}(t)+\eta_{i}(t), \quad i=1,2,
$$

with vanishing mean

$$
\left\langle\eta_{i}(t)\right\rangle=0
$$

and correlations

$$
\left\langle\eta_{i}(t) \eta_{j}\left(t^{\prime}\right)\right\rangle=S_{i j}^{\text {noise }}\left(t-t^{\prime}\right), \quad\left\langle\eta_{i}(t) z_{j}\left(t^{\prime}\right)\right\rangle=0 .
$$

In the actual experimental setup, contributions to the noise $\eta_{i}$ include vibrations of the optical components in the optical path, electronic noise in the detectors as well as shot noise.
Using Eqs. (17) and (18) and the above statistical properties of the instrumental noise, the PSD of the electric signals, defined by

$$
\left\langle z_{i}(\omega) z_{j}^{\star}\left(\omega^{\prime}\right)\right\rangle=2 \pi \delta\left(\omega-\omega^{\prime}\right) S_{i j}(\omega),
$$

can be related to the thermal PSDs $S_{i j}^{\text {th }}$ defined in Sec. III A and the noise PSDs $S_{i j}^{\text {noise, }}$

$$
S_{i j}(\omega) \equiv f_{i}(\omega) f_{j}^{\star}(\omega) \sum_{k, l=1}^{2} \epsilon_{i k} \epsilon_{j l} S_{k l}^{\mathrm{th}}(\omega)+S_{i j}^{\mathrm{noise}}(\omega) .
$$

Due to crosstalk, filter and noise characteristics, these PSDs $S_{i j}$ may dramatically differ from the thermal PSDs $S_{i j}^{\text {th }}$, in particular: (i) The cross-PSD $S_{12}$ can be dominated by $S_{11}^{\text {th }}$ or $S_{22}^{\text {th }}$ due to crosstalk, if $\epsilon_{21} S_{11}^{\text {th }} \gtrsim \epsilon_{22} S_{12}^{\text {th }}$ or $\epsilon_{12} S_{22}^{\text {th }} \gtrsim \epsilon_{11} S_{12}^{\text {th }}$, which is always true for zero crossings of $S_{12}^{\text {th }}$, (ii) although all thermal PSDs $S_{i j}^{\text {th }}(i, j=1,2)$ are real (cf. Sec. III A), the cross-PSD $S_{12}$ may have a non-vanishing imaginary part, if the filter characteristics of the two position sensing devices and electronics differ such that $\operatorname{Im}\left[f_{1} f_{2}^{\star}\right] \neq 0$, see Appendix B for details, and (iii) auto-PSDs $S_{i i}$ are generally more affected by instrumental noise than cross-PSDs, since the instrumental noise in the two channels is (almost) independent, $S_{i i}^{\text {noise }} \gg S_{i j}^{\text {noise }}, i \neq j$.

\section{B. Finite time resolution and measurement time}

Discretely sampled experimental signals form the basis of the spectral analysis and are used to estimate auto- and cross-PSDs applying a DFT on the recorded time series. The use of a DFT instead of a continuous Fourier transform introduces systematic artifacts as detailed in Appendix D. Most prominently, due to the finite sampling interval $\Delta t$ one obtains estimates for the aliased PSDs

$$
S_{i j}^{\mathrm{al} .}\left(\omega_{n}\right) \equiv \sum_{m=-\infty}^{\infty} S_{i j}\left(\omega_{n}+m \frac{2 \pi}{\Delta t}\right)
$$

at discrete frequencies

$$
\omega_{n} \equiv \frac{2 \pi n}{T},
$$

where $T=N \Delta t$ is the length of the time series and $n$ is an integer. The double-infinite sum over all integers $m$ in Eq. (23) is in practice approximated numerically using suitable lower and upper cutoffs, see Appendix D for details.

\section{RELATING EXPERIMENT AND THEORY}

In equilibrium optical tweezer experiments, the recorded signals reflect the stochastic, thermal fluctuations of the beads in the setup; periodograms calculated from experimental time series are therefore random variables, the statistical properties of which are discussed in Sec. VI A, while the explicit calculations are found in Appendix C. Based on these statistics, a maximum likelihood method is proposed in Sec. VI B, which allows to determine unknown parameters by a global fit of auto- and cross-PSDs to the periodogram values. Quantifying the deviations between fit and experimental data may serve 
to validate the underlying model as shown in Sec. VI C. The overall spectral analysis is summarized in Sec. VI D.

\section{A. Statistical properties of auto- and cross-periodograms}

The thermal motion of the beads being stochastic, the signals $z_{i}(t)$ recorded over a time window $T$, their Fourier components $z_{i}(\omega)$ as well as real and imaginary parts of the periodograms

$$
P_{i j}(n) \equiv \frac{z_{i}\left(\omega_{n}\right) z_{j}^{\star}\left(\omega_{n}\right)}{T}
$$

are stochastic quantities. Leakage effects (cf. Appendix D 1) being negligible in the present case ${ }^{9}$ as long as the window time $T$ is much larger than all relevant time scales in the system, the expectation values of real and imaginary parts of the periodograms by definition (cf. Eqs. (21) and (25)) coincide with the corresponding real and imaginary parts of the PSDs

$$
\begin{aligned}
\left\langle\operatorname{Re}\left[P_{i j}(n)\right]\right\rangle & =\operatorname{Re}\left[S_{i j}\left(\omega_{n}\right)\right], \\
\left\langle\operatorname{Im}\left[P_{i j}(n)\right]\right\rangle & =\operatorname{Im}\left[S_{i j}\left(\omega_{n}\right)\right] .
\end{aligned}
$$

However, the values of individual periodograms are broadly distributed around these expectation values as is seen from the variances of these quantities. From the explicit calculations in Appendix C resulting in Eqs. (C7) to (C9), one obtains ${ }^{8}$

$$
\begin{aligned}
& \Sigma^{2}\left[\operatorname{Re}\left[P_{i j}\right]\right]=\frac{1}{2}\left(S_{i i} S_{j j}+\operatorname{Re}\left[S_{i j}\right]^{2}-\operatorname{Im}\left[S_{i j}\right]^{2}\right), \\
& \Sigma^{2}\left[\operatorname{Im}\left[P_{i j}\right]\right]=\frac{1}{2}\left(S_{i i} S_{j j}-\operatorname{Re}\left[S_{i j}\right]^{2}+\operatorname{Im}\left[S_{i j}\right]^{2}\right),
\end{aligned}
$$

where $\Sigma^{2}[\ldots] \equiv\left\langle\ldots{ }^{2}\right\rangle-\langle\ldots\rangle^{2}$ denotes the second central moment and the frequency arguments have for simplicity been omitted. For auto-periodograms $(i=j)$, which by definition are real and positive, $\Sigma^{2}\left[\operatorname{Re}\left[P_{i i}\right]\right]=S_{i i}^{2}$ since $\operatorname{Im}\left[S_{i i}\right]$ $\equiv 0$ in Eq. (28); the statistical uncertainty thus coincides with the expectation value $S_{i i}$. More specifically, one can show that the values of the periodogram $P_{i i}$ are exponentially distributed; ${ }^{9,36}$ note that periodogram values are nonself-averaging, meaning that increasing the signal length $T$ yields periodogram estimates for a larger set of discrete frequencies and reduces leakage effects (cf. Appendix D 1), but has no influence on the periodogram statistics.

For the real and imaginary parts of the crossperiodograms $(i \neq j)$, the situation is even worse, since the statistical uncertainties will in general exceed the expectation values due to the dominance of the term $S_{i i} S_{j j}$ in Eqs. (28) and (29); in the general case where $S_{i j} \neq 0$, the form of the probability distribution functions (PDFs) for real and imaginary parts of the cross-periodograms are not simple. ${ }^{8}$

The previous statements, which are based on the use of the continuous Fourier transformation, also apply to the periodograms based on the DFT of discretely sampled time series, if the PSDs $S_{i j}$ in Eqs. (26)-(29) are replaced by their aliased equivalents $S_{i j}^{\text {al. }}$ defined in Eq. (23), see Appendix C for details.

The way to deal with the above mentioned statistical uncertainties is of course to average periodograms over $N_{\mathrm{w}}$ statistically independent time windows and (possibly) over blocks of $N_{\mathrm{b}}$ discrete, neighboring frequencies, yielding averaged periodograms

$$
\bar{P}_{i j}(n) \equiv \frac{1}{N_{\mathrm{w}} N_{\mathrm{b}}} \sum_{k=1}^{N_{\mathrm{w}}} \sum_{l} P_{i j}^{(k)}(l),
$$

where the superscript $(k)$ discriminates the periodogram values of different time windows and the index $l$ runs over the block of $N_{b}$ frequencies around $\omega_{n}$. Note that "blocking" may cause artifacts: for a single trap blocking effects are negligible as long as $N_{\mathrm{b}} \ll \kappa \mu_{0} T /(2 \pi)$, see Ref. 9 for further details. According to the central limit theorem, the statistical uncertainty $\Sigma$ of the averaged periodograms is reduced by a factor $1 / \sqrt{N_{\mathrm{w}} N_{\mathrm{b}}}$ with respect to the periodograms of individual time windows; the values of $\bar{P}_{i j}$ thus represent statistically more reliable experimental estimates of the PSD $S_{i j}$.

Clearly, not the individual values of the auto- and crossPSDs $S_{i j}$ are of interest, but the values of the parameters in the theoretical model, which yield the best agreement between theory and experimental data, namely the values of averaged auto- and cross-periodograms $\bar{P}_{i j}$ at the discrete frequencies $\omega_{n}$ resolved experimentally. A controlled way of inferring these parameters from the data taking into account the statistical aspects discussed in this section is given by the maximum likelihood method described in Sec. VI B.

A schematic summarizing the signal processing described in Sec. V and the relationship between periodograms and PSDs is found in Fig. 5.

\section{B. Maximum likelihood fits of PSDs}

The theoretical auto- and cross-PSDs $S_{i j}$ of the electric signals involve parameters both on the level of the thermal motion of the experimental object (trap strengths, particle radii, etc., cf. Secs. III and IV) as well as on the level of the signal processing in the instrument (amplification, polarization crosstalk, parasitic filtering, etc., cf. Sec. V and Appendix B). For averaged periodograms $\bar{P}_{i j}$ (Eq. (30)) calculated from experimental time series, optimal parameter values can be obtained by Bayesian inference, which thus constitutes the missing link between theory and experiment in Fig. 5. The strength of the method consists in the fact that the statistical aspects discussed in Sec. VI A are inherently taken into account.

In general, given some measured data, the most likely parameters (params) are those maximizing the conditional probability $\mathcal{P}$ (params|data). Using Bayes' theorem

$$
\mathcal{P}(\text { params } \mid \text { data })=\frac{\mathcal{P}(\text { data } \mid \text { params }) \mathcal{P}(\text { params })}{\mathcal{P}(\text { data })},
$$

and assuming a uniform prior distribution of the parameters, i.e., $\mathcal{P}$ (params) $=$ const., maximizing $\mathcal{P}$ (params $\mid$ data) turns out to be equivalent to maximizing the conditional probability $\mathcal{P}$ (data|params) of observing some data given a set of parameters. In the present case, the data consists of the ensemble of averaged periodogram vectors

$$
\overline{\boldsymbol{P}} \equiv\left(\bar{P}_{11}, \bar{P}_{22}, \operatorname{Re}\left[\bar{P}_{12}\right], \operatorname{Im}\left[\bar{P}_{12}\right]\right)^{\mathrm{T}},
$$




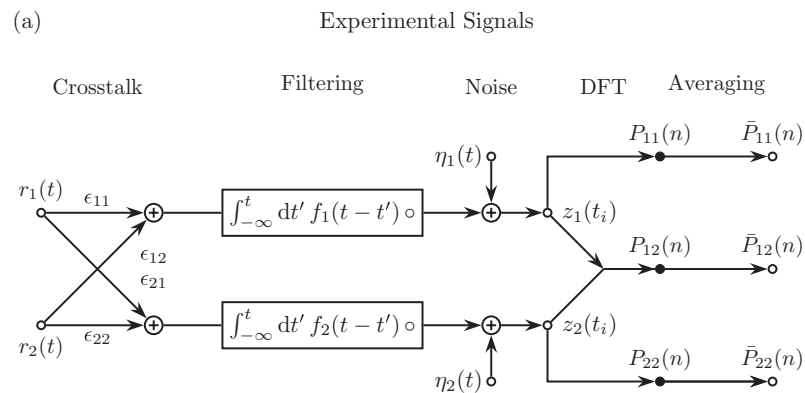

(b)

Power Spectral Densities

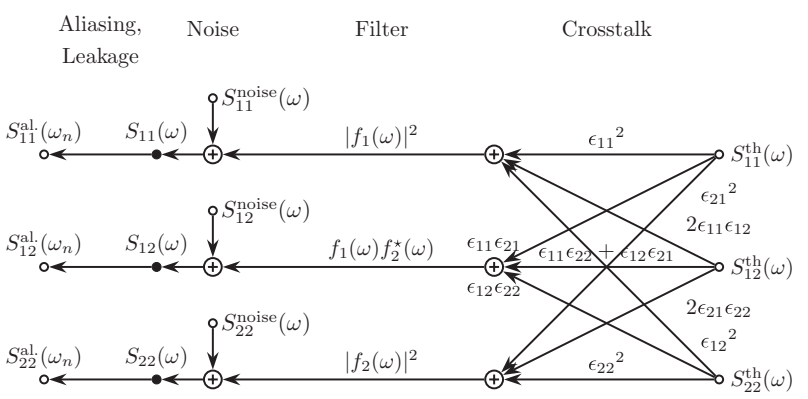

FIG. 5. Visualization of the signals in a typical dual trap optical tweezer experiment as described in Secs. V and VI A. (a) Signal processing as described by Eqs. (17) and (18); averaged periodograms $\bar{P}_{i j}$ obtained via DFT from the discretely sampled stochastic trajectories yield experimental estimates for the aliased PSDs $S_{i j}^{\text {al. }}$. (b) Relation between auto- and cross-PSDs $S_{i j}^{\text {th }}$ of the beads' thermal motion and aliased electrical PSDs $S_{i j}^{\text {all }}$ according to Eqs. (22) and (23). Unknown parameters in the PSDs can be determined via a global maximum likelihood fit to $\bar{P}_{i j}$ as explained in Sec. VI B.

at each of the $N_{\mathrm{f}}$ discrete frequencies resolved in the spectral analysis. Periodograms of distinct time windows are independent random variables drawn from a PDF with finite first and second moments given in Sec. VI A and Appendix C. According to the central limit theorem, when averaging over a large number $N_{\mathrm{w}} N_{\mathrm{b}}$ of realizations, the PDF for an averaged periodogram vector $\overline{\boldsymbol{P}}$ converges to a multivariate normal distribution characterized by the expectation vector

$$
\langle\overline{\boldsymbol{P}}\rangle=S^{\text {al. }} \equiv\left(S_{11}^{\text {al. }}, S_{22}^{\text {al. }}, \operatorname{Re}\left[S_{12}^{\text {al. }}\right], \operatorname{Im}\left[S_{12}^{\text {al. }}\right]\right)^{\mathrm{T}},
$$

and the covariance matrix resulting from the periodogram covariances calculated in Appendix C,

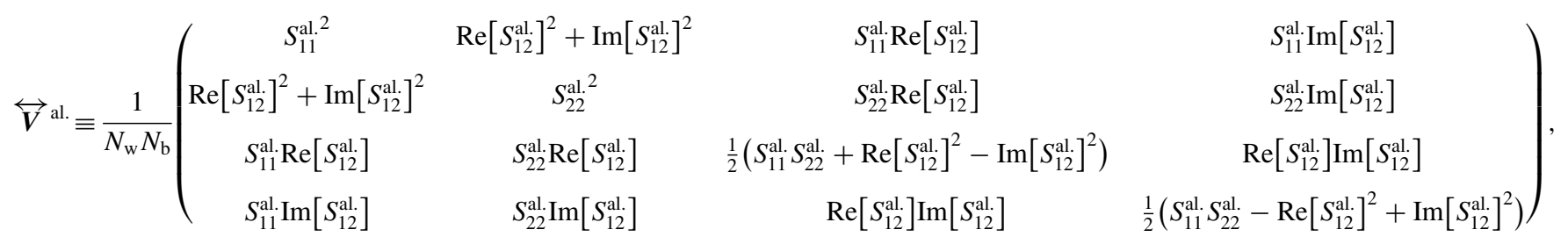

where the (discrete) frequency arguments $n$ and $\omega_{n}$ are again omitted for simplicity. Periodogram values at different frequencies being (approximately) independent, ${ }^{8}$ the overall conditional probability of observing a certain ensemble of averaged periodograms given a particular set of parameters is just the product of the $N_{\mathrm{f}}$ multivariate normal distributions

$$
\begin{aligned}
& \mathcal{P}(\text { data } \mid \text { params }) \\
& =\prod_{n}\left(4 \pi^{2} \sqrt{\operatorname{det}\left(\overleftrightarrow{\boldsymbol{V}} \text { al. }\left(\omega_{n}\right)\right)}\right)^{-1} \\
& \quad \times \exp \left(-\frac{1}{2}\left(\overline{\boldsymbol{P}}(n)-\boldsymbol{S}^{\mathrm{al} .}\left(\omega_{n}\right)\right)^{\mathrm{T}} \cdot \overleftrightarrow{\boldsymbol{V}}^{\mathrm{al}^{-1}}\left(\omega_{n}\right)\right. \\
& \left.\quad \cdot\left(\overline{\boldsymbol{P}}(n)-\boldsymbol{S}^{\mathrm{al} .}\left(\omega_{n}\right)\right)\right) .
\end{aligned}
$$

For a given ensemble of averaged periodograms, the optimal set of parameters is obtained by maximizing the above conditional probability with respect to these parameters; by reason of numerical stability, it is however preferred to minimize the negative $\log$-likelihood $-\log (\mathcal{P}$ (data|params $))$, which up to constants equals

$$
\begin{aligned}
\mathcal{F} \equiv & \sum_{n} \frac{1}{2}\left(\overline{\boldsymbol{P}}(n)-\boldsymbol{S}^{\mathrm{al} .}\left(\omega_{n}\right)\right)^{\mathrm{T}} \cdot \overleftrightarrow{\boldsymbol{V}}^{\text {al. }}{ }^{-1}\left(\omega_{n}\right) \\
& \cdot\left(\overline{\boldsymbol{P}}(n)-\boldsymbol{S}^{\mathrm{al} .}\left(\omega_{n}\right)\right)+\frac{1}{2} \log \left[\operatorname{det}\left(\overleftrightarrow{\boldsymbol{V}}^{\mathrm{al} .}\left(\omega_{n}\right)\right)\right] .
\end{aligned}
$$

The maximum likelihood method for determining the optimal set of parameters in the theoretical model consists of performing a minimization of Eq. (35) in the (multidimensional) parameter space; since the second term in Eq. (35) can be neglected if $\sqrt{N_{\mathrm{w}} N_{\mathrm{b}}(n)} \gg 1$, one is left with minimizing

$$
\begin{aligned}
\tilde{\mathcal{F}} \equiv & \frac{1}{2} \sum_{n}\left(\overline{\boldsymbol{P}}(n)-\boldsymbol{S}^{\text {al. }}\left(\omega_{n}\right)\right)^{\mathrm{T}} \cdot \overleftrightarrow{\boldsymbol{V}}^{\mathrm{al}^{-1}}\left(\omega_{n}\right) \\
& \cdot\left(\overline{\boldsymbol{P}}(n)-\boldsymbol{S}^{\mathrm{al} .}\left(\omega_{n}\right)\right),
\end{aligned}
$$

which corresponds to a standard multidimensional leastsquare fit, for which a handy implementation in $\mathrm{C} / \mathrm{C}++$ is provided by Lourakis. ${ }^{37}$

Note that in the case of one trap and thus a single signal, $V^{\text {al. }}=S_{11}^{\text {al. }}{ }^{2} /\left(N_{\mathrm{w}} N_{\mathrm{b}}\right)$ and Eq. (35) (again up to constants) 
reduces to

$$
\sum_{n} \frac{N_{\mathrm{w}} N_{\mathrm{b}}(n)}{2}\left(\frac{\bar{P}_{11}(n)}{S_{11}^{\mathrm{al}}\left(\omega_{n}\right)}-1\right)^{2}+\log \left(S_{11}^{\mathrm{al}}\left(\omega_{n}\right)\right) .
$$

This is the quantity which is commonly minimized for the calibration of single trap setups. ${ }^{9}$

\section{Deviations of fits and data}

Once Eq. (36) has been minimized numerically, the encountered minimal value

$$
\tilde{\mathcal{F}}_{\text {min }} \equiv \min _{\text {params }} \tilde{\mathcal{F}}
$$

quantifies the remaining squared deviations between averaged experimental periodograms and theoretical PSDs. The fit quality can be judged based on the sample average of these deviations per experimental observable

$$
\chi^{2} \equiv \frac{2}{N_{\overline{\boldsymbol{P}}} N_{\mathrm{f}}} \tilde{\mathcal{F}}_{\text {min }},
$$

where $N_{\mathrm{f}}$ denotes the number of discrete frequencies resolved in the spectral analysis and $N_{\bar{P}}$ is the dimension of the periodogram vector $\overline{\boldsymbol{P}}$ defined in Eq. (32). The expectation value of $\chi^{2}$ being 1 , the encountered value of $\chi^{2}$ is an indicator for the quality of the theoretical modeling of the experimental signals: both values $\chi^{2} \gg 1$ and $\chi^{2} \ll 1$ indicate deficits in the model and comparing $\chi^{2}$-values may help to discriminate between various potential models.

In some cases, it may be helpful to restrict the computation of $\tilde{\mathcal{F}}$ and $\chi^{2}$ to a subset of the observables in $\overline{\boldsymbol{P}}$ to localize the source of the deviations observed. If for example only $P_{11}$ and $\operatorname{Re}\left[P_{12}\right]$ are considered, only the first and third columns and rows in $\overleftrightarrow{\boldsymbol{V}}$ al. are taken into account and one sets $N_{\overline{\boldsymbol{P}}}=2$ in Eq. (39).

\section{Power spectral analysis in a nutshell}

Overall, the auto- and cross-power spectral analysis of optical tweezer experiments proposed in this paper involves the following procedure: (i) determine the auto- and cross-PSDs $S_{i j}^{\text {th }}$ describing the thermal fluctuations of the beads as parts of the overall experimental construct under study (Sec. III), (ii) identify the relations between bead positions and detected signals (Sec. V) and deduce their autoand cross-PSDs $S_{i j}$, (iii) perform the actual experiment and calculate averaged auto- and cross periodograms $\bar{P}_{i j}$ from the experimental time series via Eqs. (25) and (30), (iv) perform a numerical minimization of Eq. (36) to determine the optimal parameter values compatible with the data, (v) judge on the validity of the theoretical model based on the deviations between best fits and the experimental data (Sec. VI C).

In a first step, this procedure allows a precise calibration of the instrument by three consecutive calibration measurements as shown in Sec. VII; in a second step, additional unknown parameters of more complex experimental constructs, characterizing for example the viscoelastic properties of the molecular tether under study, can be determined.

\section{CALIBRATION OF DUAL TRAP OPTICAL TWEEZERS}

A series of three measurements is proposed for the calibration of a dual trap optical tweezer setup: (i) both traps empty, in the following named Cal. I, (ii) first trap occupied, second one empty (Cal. II), and (iii) second trap occupied, first one empty (Cal. III). In Cal. I, all thermal PSDs vanish, $S_{i j}^{\text {th }}=0, \forall i, j$, and therefore according to Fig. 5 the recorded periodograms $\bar{P}_{i j}$ result from the instrumental noise only. In Cal. II, only the thermal PSD $S_{11}^{\text {th }}$ is non-zero reflecting the stochastic motion of a single bead in the first optical trap ${ }^{9}$ and similarly $S_{22}^{\text {th }}$ is the only non-vanishing thermal PSD in Cal. III; explicit expressions for the thermal PSD of a single bead in a harmonic trap are for example found in Ref. 9.

Detector output voltages are recorded with $100 \mathrm{kHz}$ sampling rate over a time window of $60 \mathrm{~s}$ for each of the calibration experiments; the time series are analyzed using 6000 windows of $T=10 \mathrm{~ms}$ duration each, and blocking neighboring periodogram values resulting in blocked periodogram values at 84 distinct discrete frequencies in the range of $100 \mathrm{~Hz}$ to $50 \mathrm{kHz}$. The averaged periodograms resulting from the spectral analysis are denoted as symbols in Fig. 6, where top and bottom figures show data corresponding to the spatial direction parallel and perpendicular to the inter-trap axis, respectively.

The averaged periodograms from Cal. I shown in Fig. 6(a) reveal that the instrumental noise recorded by the two detectors is to a major extent independent, demonstrated by the fact that typical amplitudes of the cross-periodograms $\bar{P}_{12}$ are suppressed by one order of magnitude or more with respect to the auto-periodogram values $\bar{P}_{11}$ and $\bar{P}_{22}$. The functional form of the instrumental noise-PSDs $S_{i j}^{\text {noise }}$, the Fourier transformations of the noise correlation functions in Eq. (20), can be modeled as a uniform white-noise background spectrum superimposed by a $1 / f$-PSD as well as a number of spikes at well-defined frequencies; given that, according to Eq. (22), noise PSDs simply yield an additive contribution to the detected PSDs $S_{i j}$, we restrict ourselves to directly use the values of the averaged periodograms in Fig. 6(a) as reliable estimates of the aliased noise PSDs for the analysis of the subsequent experiments. Assuming stationarity and statistical independence of the time windows, the expected statistical error for the averaged auto-periodograms is in fact on the order of one percent or below, depending on the block size.

According to Fig. 5(b), one expects contributions of the thermal motion of the bead in the first trap $\left(S_{11}^{\text {th }}\right)$ and of the instrumental noise $\left(S_{i j}^{\text {noise }}\right)$ to the measured periodograms of Cal. II. The averaged periodograms $\bar{P}_{11}$ (red circles) in Fig. 6(b) in fact reflect the typical Lorentzian-like shape of $S_{11}^{\text {th }}$. Due to polarization crosstalk $\left(\epsilon_{21} \neq 0\right)$ the thermal motion of the bead is also observed in the real part of the cross-periodograms, $\operatorname{Re}\left[\bar{P}_{12}\right]$, shown as green symbols, though reduced in amplitude by a factor $\epsilon_{21} / \epsilon_{11}$ with respect to $\bar{P}_{11}$. The auto-periodogram of the second signal $\bar{P}_{22}$ (blue circles) is clearly dominated by the instrumental noise, the thermal motion of the bead introducing only slight changes with respect to Cal. I in Fig. 6(a). The imaginary parts of the cross-periodogram values, $\operatorname{Im}\left[\bar{P}_{12}\right]$ (orange symbols), result 
(a) Cal. I

(b) Cal. II

(c) Cal. III

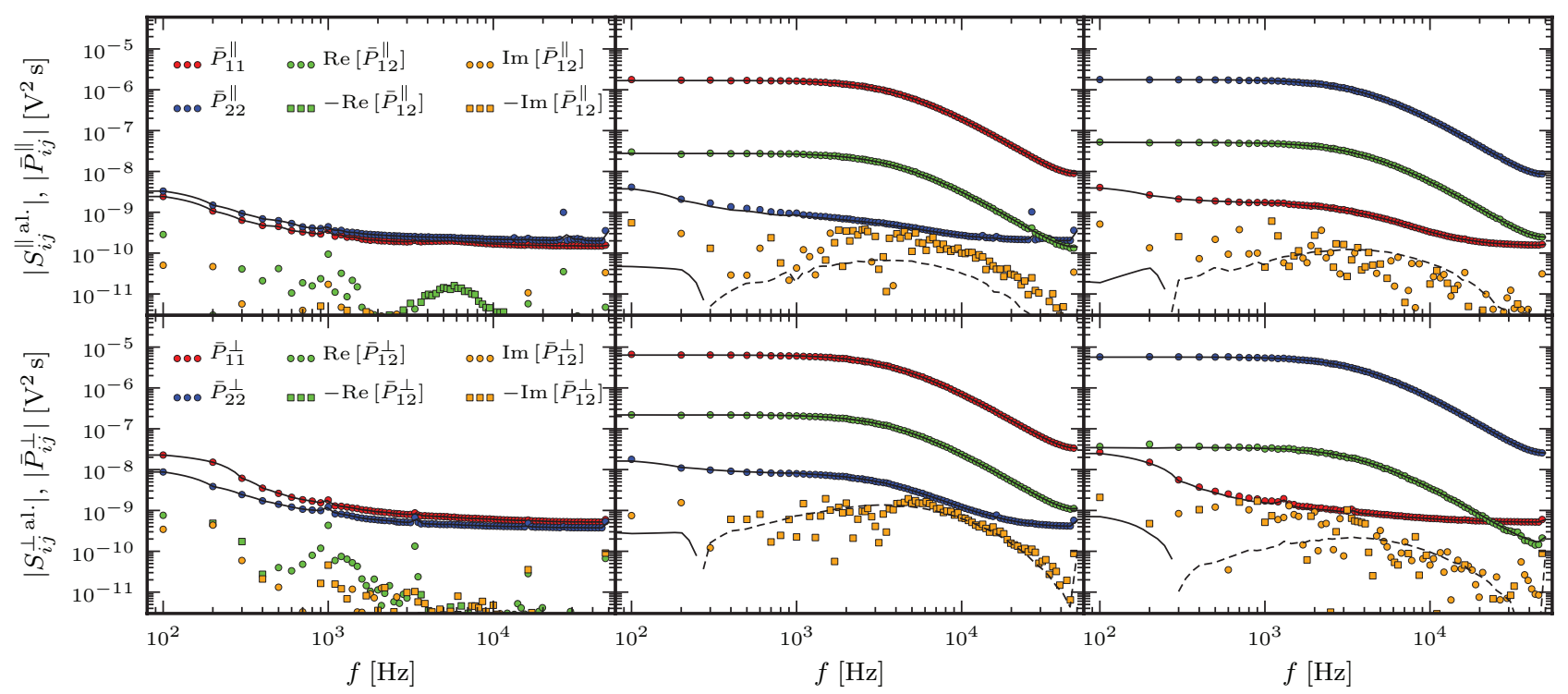

FIG. 6. Results of the three calibration measurements described in Sec. VII: (a) no trapped beads, (b) first trap occupied and second one empty, (c) vice versa. Averaged experimental periodograms $\bar{P}_{i j}^{\gamma}$ are denoted by symbols, theoretical aliased PSDs $S_{i j}^{\gamma \text { al. }}$, based on a global maximum likelihood fit to the data as described in the text, are shown as lines, where broken lines correspond to negative values of the PSDs; fit values are given in Table I. Results for the spatial coordinates parallel to the inter-trap axis $(\gamma=\|)$ are shown in the upper row, those for the perpendicular direction $(\gamma=\perp)$ in the lower one.

from an asymmetry in the signal filtering properties of the two channels as well as from the statistical uncertainty and will be discussed later. Up to this point, the undetermined parameters are the trap strength $\kappa_{1}$ in $S_{11}^{\text {th }}$, the $\mathrm{V} / \mathrm{nm}$ conversion factors $\epsilon_{11}$ and $\epsilon_{21}$ as well as parameters $\lambda_{i}$ and $\tau_{i}(i=1,2)$ reflecting the parasitic filtering properties of the two position sensing devices and entering the filter functions $f_{1}$ and $f_{2}$ (see Appendix B 1 for further details). The situation for Cal. III is analogous to the previous discussion of Cal. II except for indices 1 and 2 interchanged, adding three additional parameters $\kappa_{2}, \epsilon_{12}$, and $\epsilon_{22}$.

Since voltage amplitudes and polarization crosstalk differ for both spatial directions, we treat the corresponding data sets separately. We use $\eta=0.949 \times 10^{-3} \mathrm{~N} \mathrm{~s} / \mathrm{m}^{2}$ and $v=0.951 \times 10^{-6} \mathrm{~m}^{2} / \mathrm{s}$ for the dynamic and kinematic viscosity of water at the experimental temperature $T=22.6^{\circ} \mathrm{C}$ and the radius $a=500 \mathrm{~nm}$ given by the manufacturer for both beads. The values of the 10 parameters are determined by a global maximum likelihood fit to averaged auto- and crossperiodograms from Cal. II and III as described in Sec. VI B. Best fits to the data are shown as black lines in Fig. 6, values of the fit parameters including confidence intervals are found in Table I. As expected, the fit values for the trap stiffnesses $\kappa_{1}$ and $\kappa_{2}$ as well as for the detector properties $\left(\lambda_{1}, \lambda_{2}, \tau_{1}, \tau_{2}\right)$ for the two orthogonal spatial directions $\|$ and $\perp$ in Table I agree within error.

In Fig. 6, the agreement of experimental data and fits for the auto-PSDs $S_{11}$ and $S_{22}$ and the real part of the cross-PSD, $\operatorname{Re}\left[S_{12}\right]$, is excellent over the entire range of frequencies for both Cal. II and III. As is seen from Table I, the sample average of the (normalized) squared deviations of the data from the PSD values $\chi^{2}$ defined in Eq. (39) is relatively large compared to the expectation value of 1 , the reasons for which most likely are: (i) differences in the instrumental noise $S_{i j}^{\text {noise }}$ in
Cal. I compared to Cal. II or III, (ii) other non-stationary effects in the experimental setup, which are intrinsically difficult to model and to quantify, and (iii) an inconsistency between Cal. II and III concerning the imaginary parts of the cross-periodograms $I P_{12}$, which is discussed at the end of this section. In fact, when restricting the computation of $\chi^{2}$ to the quantities which are less sensitive to the above mentioned effects, i.e., $P_{11}$ and $\operatorname{Re}\left[P_{12}\right]$ in Cal. II as well as $P_{22}$ and $\operatorname{Re}\left[P_{12}\right]$ in Cal. III, one obtains reasonable values for the squared deviations denoted by $\chi_{\text {red }}^{2}$ in Table I.

Once the setup is calibrated as described, all instrumental parameters are set and the link between electric PSDs $S_{i j}$ and the thermal PSDs $S_{i j}^{\text {th }}$ is thus established, as is seen from the signal processing summarized in Fig. 5(b). For more

TABLE I. Calibration results: Best fit parameters obtained from global maximum likelihood fits to the averaged periodograms in Fig. 6 as described in Sec. VII.

\begin{tabular}{lcccc}
\hline \hline Quantity & Symbol & Values $\|$ & Values $\perp$ & Units \\
\hline Trap strength & $\kappa_{1}$ & $0.21 \pm 0.021$ & $0.21 \pm 0.007$ & $\mathrm{pN} / \mathrm{nm}$ \\
& $\kappa_{2}$ & $0.21 \pm 0.018$ & $0.20 \pm 0.012$ & $\mathrm{pN} / \mathrm{nm}$ \\
Amplitudes & $\epsilon_{11}$ & $32 \pm 1.1$ & $61 \pm 1.0$ & $\mathrm{mV} / \mathrm{nm}$ \\
& $\epsilon_{12}$ & $0.93 \pm 0.034$ & $0.34 \pm 0.021$ & $\mathrm{mV} / \mathrm{nm}$ \\
& $\epsilon_{21}$ & $0.52 \pm 0.025$ & $2.10 \pm 0.037$ & $\mathrm{mV} / \mathrm{nm}$ \\
& $\epsilon_{22}$ & $32 \pm 1.2$ & $56 \pm 1.0$ & $\mathrm{mV} / \mathrm{nm}$ \\
Pol. crosstalk & $\epsilon_{12} / \epsilon_{11}$ & 2.9 & 0.56 & $\%$ \\
& $\epsilon_{21} / \epsilon_{22}$ & 1.6 & 3.7 & $\%$ \\
Fraction of & $\lambda_{1}$ & $0.6 \pm 0.054$ & $0.63 \pm 0.038$ & unitless \\
fast electrons & $\lambda_{2}$ & $0.6 \pm 0.040$ & $0.61 \pm 0.023$ & unitless \\
Electr. relax. & $\tau_{1}$ & $5.7 \pm 1.5$ & $5.5 \pm 1.0$ & $\mu \mathrm{s}$ \\
Time scale & $\tau_{2}$ & $6.1 \pm 1.5$ & $6.6 \pm 0.8$ & $\mu \mathrm{s}$ \\
Normalized sq. & $\chi^{2}$ & 18 & 8.7 & unitless \\
deviations & $\chi_{\text {red }}^{2}$ & 1.8 & 2.1 & unitless \\
\hline \hline
\end{tabular}


complicated experimental constructs, e.g., single molecules tethered to the beads, additional unknown parameters characterizing the viscoelastic properties of the molecule under study and entering the thermal PSDs $S_{i j}^{\text {th }}$ of the overall experimental construct, ${ }^{7}$ can in a second step be determined from the measured auto- and cross-periodograms $\bar{P}_{i j}$ employing the same maximum likelihood fitting method. Here, we demonstrate in Sec. VIII that-without additional adjustable parameters - the periodograms recorded for two hydrodynamically interacting beads are in excellent agreement with the theoretical predictions over the entire resolved range of frequencies and for different trap separations; we thereby demonstrate the accuracy of the calibration method and the influence of the frequency dependence of $\mathrm{HI}$ discussed in Sec. IV on the PSDs.

Finally, some additional comments on the calibration spectra and the fit results:

- The radii of micron-sized beads used in optical tweezer experiments may vary from one bead to another due to finite sample variance. Determining the bead radii using a standard approach ${ }^{12}$ involves a low-frequency oscillation of the piezo stage and adds the two parameters $a_{1}$ and $a_{2}$ to be determined when calibrating of the instrument. Based on the peak amplitudes of $\bar{P}_{11}$ in Cal. II and $\bar{P}_{22}$ in Cal. III at the driving frequency of $30 \mathrm{~Hz}$ (data not shown), we found bead radii $500 \pm 5 \mathrm{~nm}$ in perfect agreement with the manufacturer's specifications (cf. Sec. II). We therefore choose a fixed bead ra$\operatorname{dius} a_{1}=a_{2}=a=500 \mathrm{~nm}$ in all our modeling. Note that the oscillation of the piezo stage also induces a periodic signal in the "empty" channel ( 2 in Cal. II and 1 in Cal. III), the amplitude and relative phase of which is less straightforward to interpret; values of the crossperiodogram and the auto-periodogram of the empty channel at the driving frequency are therefore not considered when determining $a_{1}$ and $a_{2}$. In asymmetric setups, the bead masses $m_{1}$ and $m_{2}$ in Eq. (7) would have to be adjusted and our common radius $a$ in the expressions for the self-mobilities (Eqs. (10) and (12)) would have to be replaced by $a_{1}$ and $a_{2}$, respectively; expressions for the corresponding cross-mobilities replacing Eq. (13) are found in Ref. 28.

- As is clearly seen from Table I, the polarization crosstalk amplitudes in the two channels and for the two spatial directions differ considerably ranging from $0.5 \%$ to $3.7 \%$. Moreover, the importance of crosstalk is larger in channel 1 for the $\|$-direction, while it is dominant in channel 2 for the $\perp$-direction. This observed asymmetry is presumably a consequence of the orthogonally polarized light in the two traps and the fact that the two spatial directions are detected in different layers of our position sensing devices; see the supplementary material ${ }^{38}$ for further details.

- According to our model for the signal processing in Sec. V, the ratio of real and imaginary parts of the cross-PSDs results from the filtering properties in the two channels and is given by $\operatorname{Re}\left[f_{1} f_{2}^{\star}\right] / \operatorname{Im}\left[f_{1} f_{2}^{\star}\right]$ and thus independent of the thermal PSDs $S_{i j}^{\text {th }}$, which are purely real (cf. Sec. III A). In contrast, the ratio of real and imaginary parts of the cross-periodogram values in Fig. 6-given by the distance of the periodogram values in the logarithmic plots-differ for Cal. II and Cal. III. The filtering properties of the detectors thus seem to slightly differ depending on which of the traps is occupied and which one is empty. Separate maximum likelihood fits to the data of Cal. II and Cal. III yielded marginally better fits (not shown), the fit values however remained essentially unchanged compared to the results of the global fit to both experimental data sets in Table I.

\section{DISCUSSION}

The experimental setup being calibrated as described in Sec. VII, we now compare theoretical PSDs resulting from Eqs. (8), (22), and (23) and using the fit parameters in Table I to averaged, experimental periodograms for two hydrodynamically interacting beads fluctuating in their respective laser traps; for trap separations $R$ ranging from 10 down to $1.5 \mu \mathrm{m}$, averaged periodograms are calculated from the four voltage time series $\left(z_{1, \|}, z_{2, \|}, z_{1, \perp}, z_{2, \perp}\right)$ with an overall duration of $30 \mathrm{~s}$ by analyzing time windows of $T=10 \mathrm{~ms}$ length each. The importance of hydrodynamic retardation effects are highlighted in Sec. VIII A, while the influence of instrumental effects, i.e., polarization crosstalk, (parasitic) filtering and instrumental noise, and of finite statistics is discussed in Sec. VIII B.

\section{A. Retarded vs. instantaneous hydrodynamics}

Theoretical PSDs based on frequency-dependent HI between finite-sized beads described by Eq. (13) (black lines) as well as averaged periodograms from experimental data (colored symbols) are shown for various distances $R$ between the trap centers and for motion parallel and perpendicular to the inter-trap axis in Figs. 7 and 8, respectively. Both auto-PSDs $S_{11}$ and $S_{22}$ and, in particular, the cross-PSD $S_{12}$ are affected by the varying strength of $\mathrm{HI}$ with changing trap separation or spatial direction; over the entire resolved frequency range of $100 \mathrm{~Hz}-50 \mathrm{kHz}$, excellent agreement between theoretical predictions and experimental data is observed. Note that no additional fit parameters are used in Figs. 7 and 8, demonstrating the accuracy of both the modeling of the signal processing in Sec. V as well as of the instrumental calibration in Sec. VII.

To stress the importance of the frequency dependence of HI discussed in Sec. IV B, we also plot theoretical predictions based on instantaneous $\mathrm{HI}$ described by the components of the Rotne-Prager tensor (Eq. (14)) as light blue lines in Figs. 7 and 8: the comparison reveals that the frequency dependence of the cross-mobilities shown in Fig. 4 arising from the finite time of vortex propagation in the fluid is essential for a quantitative match of theory and experiment. As expected, the influence of the frequency dependence is more prominent at large separations than at smaller ones, where the idealized picture of instantaneous HI constitutes a better, though still approximate description (cf. Sec. IV B). 


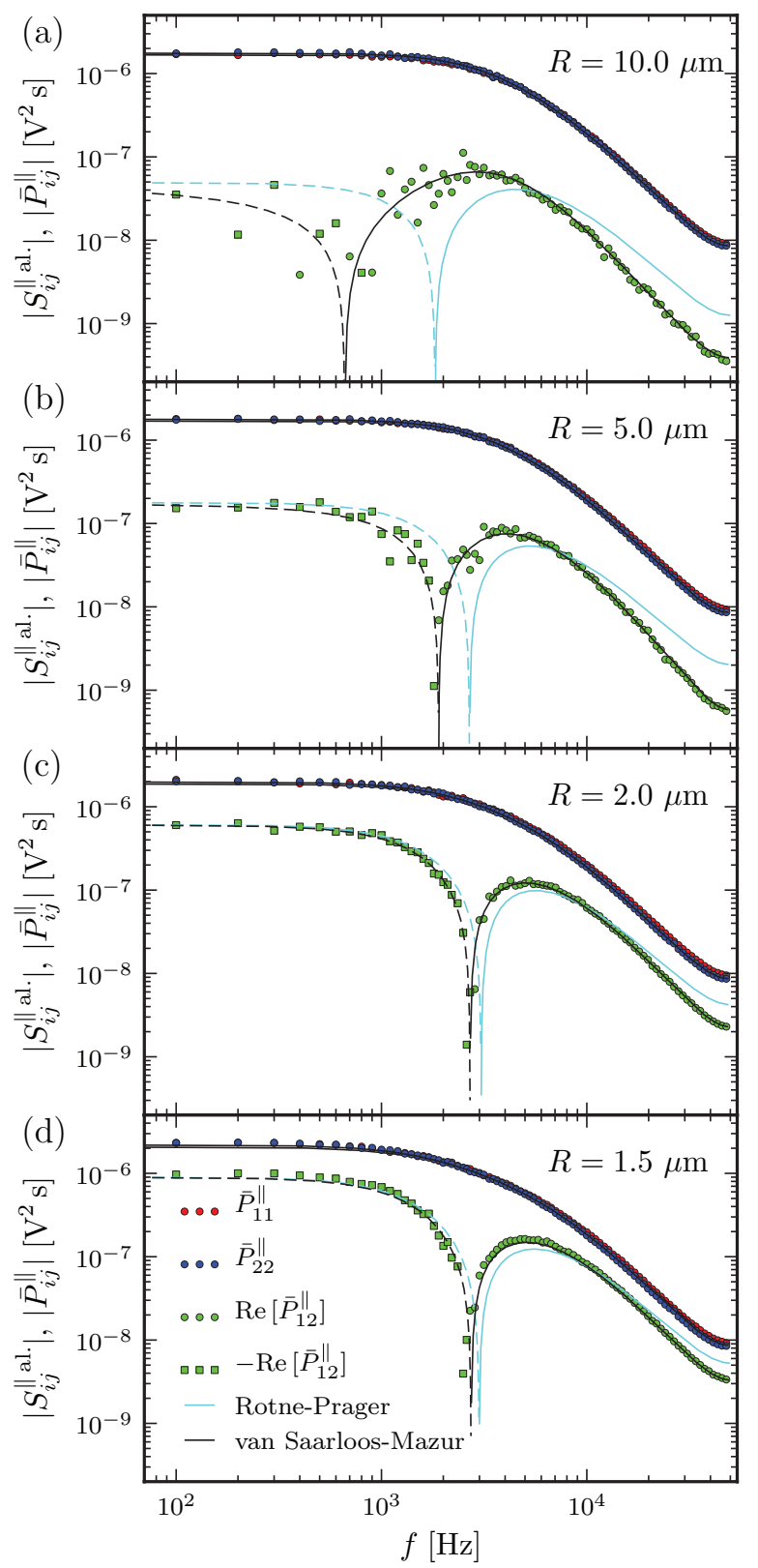

FIG. 7. Auto- and cross-PSDs of the fluctuations parallel to the inter-trap axis for various trap separations $R$ : Symbols denote averaged experimental periodograms $\bar{P}_{i j}^{\|}$, lines the theoretical predictions for the aliased PSDs $S_{i j}^{\| \text {al. }}$ according to Eqs. (22) and (23): black lines include the full frequency dependence of HI (Eq. (13)), light blue lines correspond to instantaneous HI (Eq. (14)). Positive values of the averaged experimental periodograms are denoted by circles, negative ones by squares; similarly, solid and broken lines, respectively, denote positive and negative PSD values.

Remaining deviations between theoretically predicted and recorded spectra are mainly attributed to uncertainties in the calibration fit parameters (see Table I), non-stationary instrumental effects and slight dependencies of the system parameters on the trap configuration. In particular, the signal amplitude $\epsilon_{11}$ of our mobile trap was found to vary within some percent when the trap is displaced over length scales of several $\mu \mathrm{m}$ (data not shown). If a quantitative evaluation of the spectra is intended, it is therefore recommended to perform the calibration measurements in a similar configuration as the actual experiment is done. In addition, at high frequencies systematic deviations may result from limitations in the

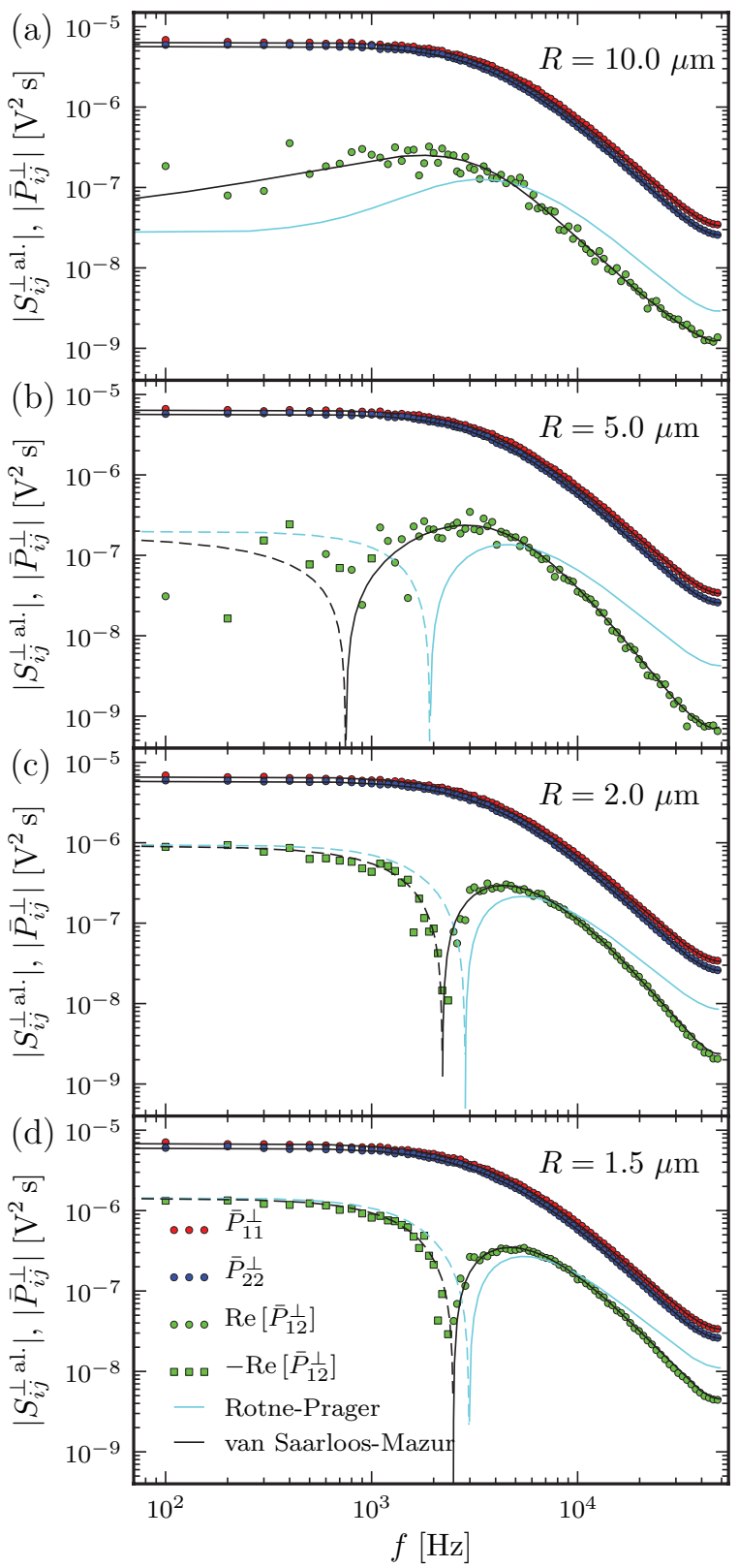

FIG. 8. Same as Fig. 7 but for the spatial component perpendicular $(\perp)$ to the inter-trap axis.

model, e.g., for the parasitic filtering covered in Appendix B 1 , and for separations $R \lesssim 3 a$, the uncertainty in the absolute distance $R$ and the neglect of higher order corrections for selfand cross-mobilities ${ }^{32}$ may play a role. Finally, alignment inaccuracies of the instrument (cf. Sec. III B) or crosstalk between the spatial directions within each of the position sensing devices ${ }^{9}$ may be responsible for residual deviations; both effects have been neglected in the present analysis, but can in principle be accounted for using the full threedimensional theory (Sec. III B) and a refined model for the signal processing involving all four recorded signals (Sec. V).

\section{B. Instrumental effects and finite statistics}

As schematically indicated in Fig. 5(b), the electric PSDs $S_{i j}$ deviate from the thermal PSDs of the beads $S_{i j}^{\text {th }}$ due to three instrumental effects: polarization crosstalk, 


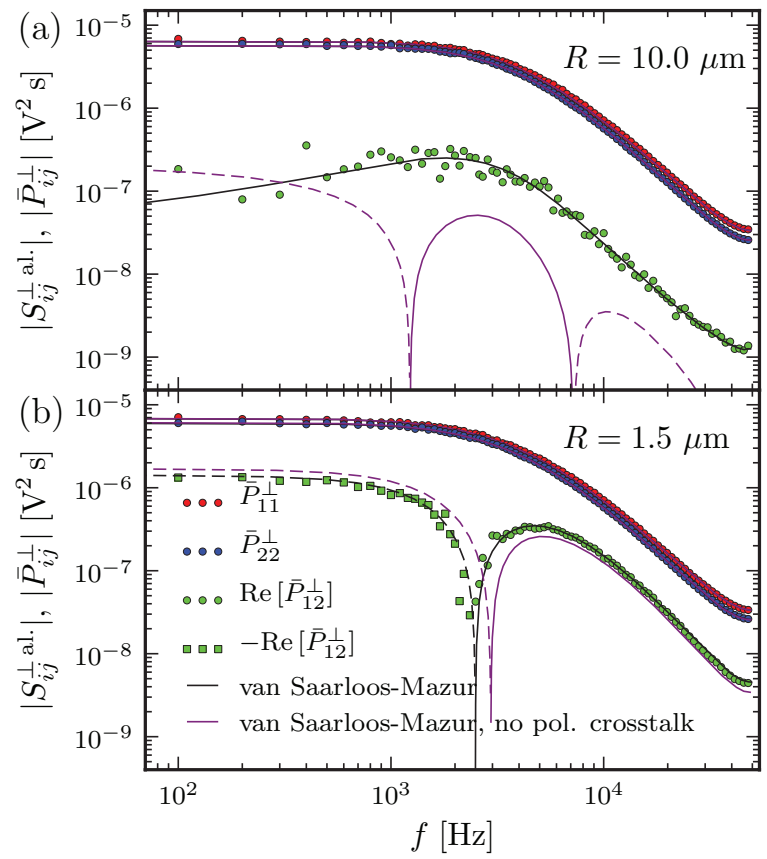

FIG. 9. Influence of polarization crosstalk: Theoretical PSDs accounting for polarization crosstalk are shown as black lines (same as in Fig. 8), theoretical PSDs neglecting polarization crosstalk are denoted as purple lines; since auto-PSDs are only marginally affected by polarization crosstalk, black and purple lines for $S_{11}$ and $S_{22}$ overlap. Symbols denote experimental data (same as in Fig. 8).

(parasitic) filtering and instrumental noise. Their influence on the calibration and hydrodynamic spectra in Figs. 6-8 is discussed in the following.

The importance of polarization crosstalk for the calibration process was covered in Sec. VII; its influence on the PSDs of two hydrodynamically interacting beads is illustrated by comparing experimental data and theoretical curves from Fig. 8 to theoretical predictions for the PSDs, which do not account for crosstalk setting $\epsilon_{12}=\epsilon_{21}=0$. The corresponding PSDs are shown as purple lines in Fig. 9 together with the original data and curves of Fig. 8. For a trap separation $R=10 \mu \mathrm{m}$ (Fig. 9(a)), where due to weak HI the thermal cross-PSD $S_{12}^{\text {th }}$ is small in amplitude compared to the thermal auto-PSDs $S_{11}^{\text {th }}$ and $S_{22}^{\text {th }}$, the observed cross-PSD of the electric signals $S_{12}$ is dominated by the thermal auto-PSDs, which couple in due to polarization-crosstalk; experimental data is consequently ill-represented when polarization crosstalk is neglected, as shown in Fig. 9(a). For smaller trap separations ( $R=1.5 \mu \mathrm{m}$ in Fig. 9(b)), where HI are stronger, the frequency dependence of the thermal cross-PSD $S_{12}^{\text {th }}$ is better reflected in $S_{12}$, though including polarization crosstalk remains necessary for a quantitative agreement between theory and experimental data. As expected, polarization crosstalk affects the auto-PSDs to a minor extent only. We conclude that a quantitative analysis of experimental (cross-)periodograms requires accounting for crosstalk to disentangle the contributions of thermal auto- and cross-PSDs.

The effects of parasitic filtering of position sensing devices (cf. Appendix B 1) on the auto-periodograms measured in single trap optical tweezer experiments has been discussed in detail before. ${ }^{11,35,39}$ For dual trap setups, deviations in the filter properties of the two devices have as additional consequence a non-vanishing imaginary part of the cross-PSD, $\operatorname{Im}\left[S_{12}\right]$, as discussed in Sec. V A and exemplified in Appendix B 1 .

The influence of instrumental noise is seen in the calibration measurements of Cal. II and III in Figs. 6(b) and 6(c); the discussion in this paragraph focuses on Cal. II, but equally applies to Cal. III with indices 1 and 2 interchanged. In the absence of noise, according to the schematic in Fig. 5(b), one expects roughly equivalent shapes for $\bar{P}_{11}, \operatorname{Re}\left[\bar{P}_{12}\right]$, and $\bar{P}_{22}$, slight differences being attributed to the individual filter functions $f_{1}$ and $f_{2}$ and the relative magnitudes being set by the ratio $\epsilon_{11} / \epsilon_{21} \gg 1$. However, the averaged periodogram values $\bar{P}_{22}$ are mostly dominated by the instrumental noise, since $S_{22}^{\text {noise }} \gtrsim \epsilon_{21}^{2} S_{11}^{\text {th }}$.

Finally, some comments on the statistical uncertainty in the data: In Figs. 6(b) and 6(c), one observes a noticeable larger scatter of the cross-periodogram values $\operatorname{Re}\left[\bar{P}_{12}\right]$ around the theoretical estimate $\operatorname{Re}\left[S_{12}\right]$ compared to the autoperiodogram values. This effect is best seen for large frequencies in the bottom panel of Fig. 6(c) corresponding to the $\perp$ direction, where the polarization crosstalk is the weakest. According to the periodogram statistics discussed in Sec. VI A, the relative statistical uncertainty of $\operatorname{Re}\left[P_{12}\right]$ is

$$
\frac{\sqrt{\Sigma^{2}\left[\operatorname{Re}\left[P_{12}\right]\right]}}{\operatorname{Re}\left[S_{12}\right]} \approx \sqrt{1+\frac{S_{11}^{\text {noise }}}{2 \epsilon_{12}^{2} S_{22}^{\text {th }}}}
$$

which exceeds the relative statistical uncertainty of autoperiodogram values (=1, cf. Sec. VI A), whenever the instrumental noise in the "empty" signal dominates over the contribution of the thermal bead motion. The instrumental noise in the two signals $z_{1}$ and $z_{2}$ being (mostly) independent, the expectation value $\left\langle\operatorname{Re}\left[\bar{P}_{12}\right]\right\rangle=\operatorname{Re}\left[S_{12}\right]$ are relatively insensitive to instrumental noise, the statistical uncertainty in the crossperiodogram values is however increased. For the case of two hydrodynamically interacting beads, the contribution of noise to the observed PSDs is rather small; taking into account instrumental noise is thus in the first place important to account for the increased statistical uncertainty of cross-periodogram values when calibrating the experimental setup as described in Sec. VII.

The averaged periodograms $\bar{P}_{i j}$ shown in the Figs. 6-9 result from the spectral analysis of at least 3000 signal windows and from blocking periodogram values corresponding to neighboring frequencies; for independent samples, the statistical uncertainty of the averaged periodograms is thus reduced by a factor $\leq 1 / \sqrt{3000} \sim 0.02$ compared to the bare periodograms. Nevertheless, for the cross-periodograms the statistical uncertainty still may be comparable or even exceed the magnitude of the expectation value (cf. Sec. VI A). As an example, the periodogram values $\operatorname{Re}\left[\bar{P}_{12}\right]$ considerably scatter around the theoretical predictions $\operatorname{Re}\left[S_{12}\right]$ in the top panels of Figs. 7 and 8. If the statistical uncertainty exceeds the magnitude of the expectation value, plotting the absolute periodogram values on a logarithmic scale leads to a spurious, smooth frequency dependence, which is a signature of the statistical uncertainty only and which can easily be misinterpreted (see Appendix E for further details). Such a spurious 
behavior is for example observed for the periodogram values $\operatorname{Im}\left[\bar{P}_{12}\right]$ in Cal. III shown in Fig. 6(c).

The statistical aspects discussed in the previous two paragraphs are rather subtle; it is therefore recommended to fit auto- and cross-periodograms simultaneously rather than independently by applying the Bayesian inference fitting method presented in Sec. VI B, which inherently accounts for the statistical properties of auto- and cross-periodograms and which was used within this manuscript for the calibration of a dual trap optical tweezer instrument.

\section{CONCLUSIONS}

In summary, we have presented a theoretical description of the signal processing in a typical dual trap optical tweezer experiment, and have proposed a maximum-likelihood method based on the statistical properties of auto- and cross-periodograms for the power spectral analysis of the experimentally recorded fluctuations. We have shown how-in a first calibration step - the method can be used to determine the instrumental parameters involved in the signal processing as well as the optical trapping strengths by combining data from three consecutive measurements. Using two unconnected, but hydrodynamically interacting beads trapped in the respective laser potentials as simple model system, we could demonstrate a number of different aspects including: (i) the frequency-dependence of the hydrodynamic coupling arising from the finite time of vortex diffusion in the viscous fluid, (ii) the influence of crosstalk, (parasitic) filtering and instrumental noise on the recorded periodograms, and (iii) potential caveats in the interpretation of experimental data due to finite statistics. We expect the present Bayesian inference method paired with dynamic (de-)convolution theory ${ }^{7}$ to enable experimentalists in a second step to recover the viscoelastic force response characteristics of the molecule of study from the measured equilibrium fluctuations of the overall experimental construct, e.g., the molecule tethered via handles to the two beads trapped in their respective laser foci.

\section{ACKNOWLEDGMENTS}

The authors thank M. Hinczewski, J.-C. Meiners and E. Schäffer for stimulating discussions and helpful comments. Financial support from the DFG (SFB 863), from the Elitenetzwerk Bayern in the framework of CompInt (Y.v.H.), and from the Nanosystems Initiative Munich (A.M., B.P., M.R.) is acknowledged.

\section{APPENDIX A: NOTATION}

An overview of frequently used symbols is found in Table II. Throughout, we use the following definition of the Fourier transformation $\mathcal{F}$ and its inverse $\mathcal{F}^{-1}$ for a general function $f(t)$

$$
\begin{aligned}
f(\omega) & =\mathcal{F}[f(t)] \equiv \int_{-\infty}^{\infty} \mathrm{d} t f(t) e^{\mathrm{i} \omega t}, \\
f(t) & =\mathcal{F}^{-1}[f(\omega)] \equiv \frac{1}{2 \pi} \int_{-\infty}^{\infty} \mathrm{d} \omega f(\omega) e^{-\mathrm{i} \omega t} .
\end{aligned}
$$

TABLE II. Overview of the notation used throughout the manuscript; suband super-scripts $\gamma$ are omitted in the text, if the discussion equally applies to all spatial directions.

\begin{tabular}{lcc}
\hline \hline Quantity & Symbol & Definition \\
\hline Spatial direction & $\gamma$ & $=o, \|, \perp$ \\
Bead/Signal indices & $i, j$ & $=1,2$ \\
Bead coordinate & $r_{i}$ & \\
Linear response function & $J_{i j}$ & Eq. (5) \\
Thermal correlation function / PSD & $S_{i j}^{\text {th }}$ & Eqs. (1), (3), and (4) \\
(Virtual) filter function & $f_{i}$ & Eq. (B9) \\
Electric signal & $z_{i}$ & Eqs. (17) and (18) \\
Electric PSD & $S_{i j}$ & Eq. (22) \\
Electric DFT component & $Z_{i}$ & Eq. (D1) \\
Sampling interval & $\Delta t$ & \\
Sampling rate & $f_{\text {sampl }}$ & $=1 / \Delta t$ \\
Nyquist frequency & $f_{\mathrm{Nyq}}$ & $=f_{\text {sampl }} / 2$ \\
No. of data points per time window & $N$ & \\
Duration of a time window & $T$ & $=N \Delta t$ \\
Periodogram & $P_{i j}$ & Eq. (25) \\
No. of independent time windows & $N_{\mathrm{w}}$ & \\
No. of discr. frequencies per block & $N_{\mathrm{b}}$ & \\
No. of blocked frequencies resolved & $N_{\mathrm{f}}$ & Eq. (30) \\
Averaged periodogram & $\bar{P}_{i j}$ & \\
\hline \hline
\end{tabular}

Functions in time and frequency domain are denoted by the same variables. Using the above convention the convolution theorem takes the form

$$
\begin{gathered}
\mathcal{F}[f * g(t)]=f(\omega) g(\omega), \\
\mathcal{F}[f(t) g(t)]=\frac{1}{2 \pi} f * g(\omega),
\end{gathered}
$$

where the convolution between the two functions $f$ and $g$ is defined as

$$
f * g(t) \equiv \int_{-\infty}^{\infty} \mathrm{d} t^{\prime} f\left(t^{\prime}\right) g\left(t-t^{\prime}\right)
$$

\section{APPENDIX B: POSSIBLE FORMS OF FILTERING}

Signal filtering can occur due to a number of different reasons, some of which are discussed in the following. Subsequent application of various filters corresponds to the iterative convolution of the original signal with the various filter kernels in time domain or, more conveniently, to the multiplication with the product of transfer functions in frequency space (Eq. (A2)).

\section{Filtering of the position sensing device}

Due to the transparency of silicon to the employed laser light of $1064 \mathrm{~nm}$ wavelength, the electric signal produced by the position sensing device differs from the actual dynamics of the centroid of the laser spot intensity. ${ }^{35,39}$ This parasitic or "virtual" filtering can be modeled ${ }^{11,39}$ via a transfer function

$$
f_{i}^{\mathrm{pf}}(\omega)=\lambda_{i}+\mathrm{i} \frac{1-\lambda_{i}}{\mathrm{i}+\omega \tau_{i}}, \quad i=1,2,
$$

where $\lambda_{i}$ is the fraction of fast (instantaneously detected) electrons and $\tau_{i}$ is the typical relaxation timescale of the slow 
electron fraction in channel $i$. PSDs thus are modified by factors

$$
\begin{aligned}
f_{i}^{\mathrm{pf}}(\omega) f_{j}^{\mathrm{pf}^{\star}}(\omega)= & \lambda_{i} \lambda_{j}+\frac{1-\lambda_{i} \lambda_{j}}{1+\mathrm{i} \omega\left(\tau_{j}-\tau_{i}\right)+\tau_{i} \tau_{j} \omega^{2}} \\
& +\mathrm{i} \omega \frac{\lambda_{j} \tau_{j}\left(1-\lambda_{i}\right)-\lambda_{i} \tau_{i}\left(1-\lambda_{j}\right)}{1+\mathrm{i} \omega\left(\tau_{j}-\tau_{i}\right)+\tau_{i} \tau_{j} \omega^{2}},
\end{aligned}
$$

which for $i=j$ reduces to

$$
\left|f_{i}^{\mathrm{pf}}(\omega)\right|^{2}=\lambda_{i}^{2}+\frac{1-\lambda_{i}^{2}}{1+\left(\omega \tau_{i}\right)^{2}},
$$

which is purely real. In contrast, even slight asymmetries in the filtering properties of the two detectors result in a nonvanishing imaginary part of $f_{1}^{\mathrm{pf}^{\mathrm{f}}} f_{2}^{\mathrm{pf}^{\star}}$ as discussed in Sec. VII, see Fig. 10(a) for typical frequency dependences.

\section{Electric filter}

The amplification device used in our setup acts as eighth order Butterworth filter with $f_{3 \mathrm{~dB}}=200 \mathrm{kHz}$ corresponding to transfer functions

$$
f_{i}^{\mathrm{B} 8}(\omega)=\frac{1}{B_{8}\left(\mathrm{i} \omega /\left(2 \pi f_{3 \mathrm{~dB}}\right)\right)},
$$

with $B_{8}$ being the eighth order Butterworth polynomial, such that

$$
f_{i}^{\mathrm{B} 8}(\omega) f_{j}^{\mathrm{B} 8^{\star}}(\omega)=\frac{1}{1+\left(\omega /\left(2 \pi f_{3 \mathrm{~dB}}\right)\right)^{16}} .
$$

In Fig. 10(b), Butterworth filters are compared to first-order low-pass filters, for which

$$
f_{i}^{\mathrm{lp}}(\omega) f_{j}^{\mathrm{p}^{\star}}(\omega)=\frac{1}{1+\left(\omega /\left(2 \pi f_{3 \mathrm{~dB}}\right)\right)^{2}} .
$$

\section{Temporal averaging}

Temporal averaging represents a form of low-pass filtering: for uniform averaging of a signal over a time window $\tau_{\mathrm{av}}$, i.e., a filter kernel

$$
f_{i}^{\mathrm{av}}(t)=\frac{1}{\tau_{\mathrm{av}}} \Theta\left(-t+\tau_{\mathrm{av}}\right) \Theta(t),
$$

where $\Theta$ denotes the Heaviside step function, one obtains

$$
f_{i}^{\mathrm{av}}(\omega) f_{i}^{\mathrm{av} \star}(\omega)=\left(\frac{2}{\omega \tau_{\mathrm{av}}} \sin \left(\frac{\omega \tau_{\mathrm{av}}}{2}\right)\right)^{2},
$$

in frequency domain. In consequence, PSDs of the timeaveraged signals are reduced in amplitude compared to unfiltered PSDs for frequencies $\omega \gtrsim 1 / \tau_{\mathrm{av}}$ as is shown in Fig. 10(c).

\section{Time delay between channels}

The temporal shift of a signal by $\tau_{\text {delay }, i}$ corresponds to

$$
f_{i}^{\text {del }}(t)=\delta\left(t-\tau_{\text {delay }, i}\right),
$$

and thus translates into

$$
f_{i}^{\text {del }}(\omega)=\exp \left(\mathrm{i} \omega \tau_{\mathrm{del}, i}\right) .
$$

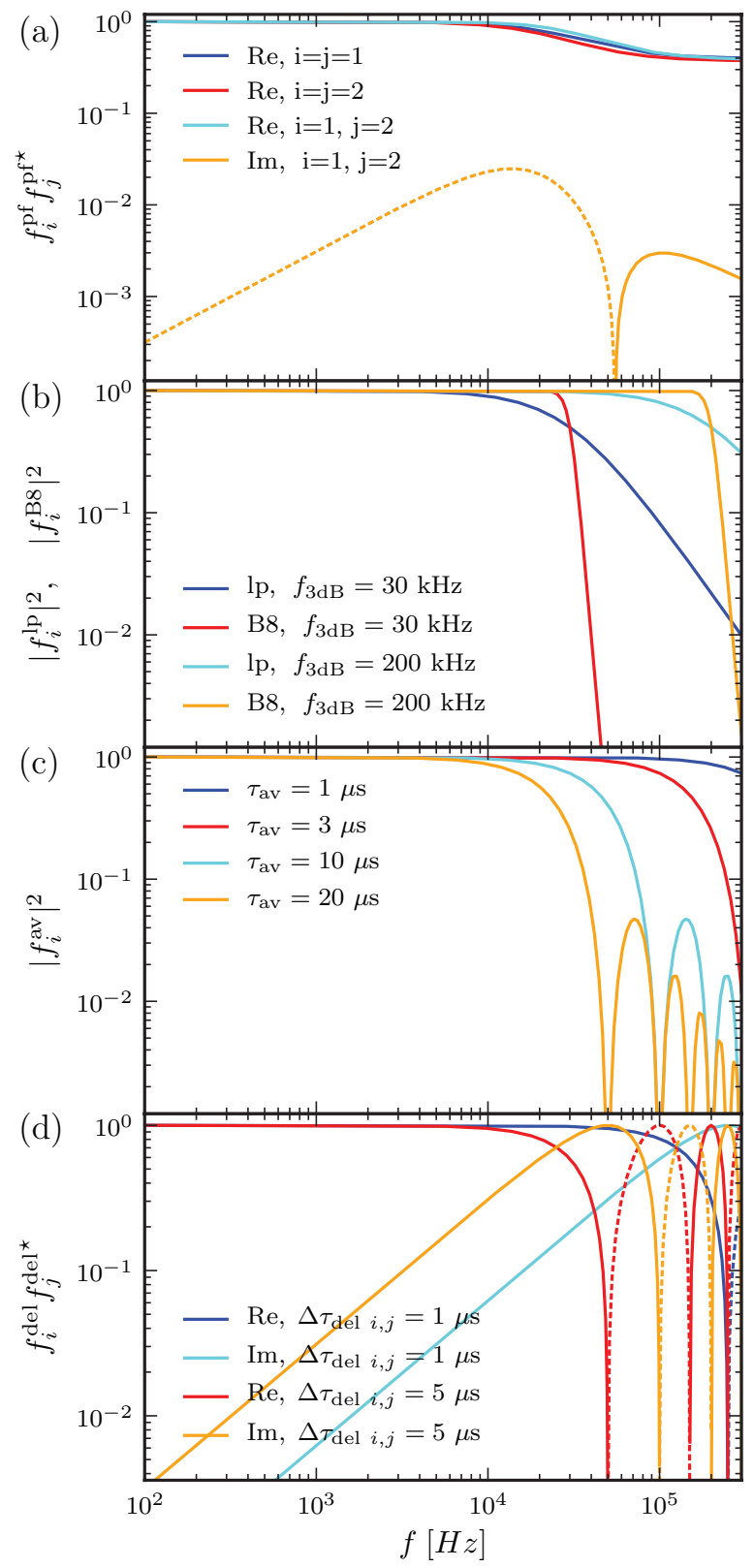

FIG. 10. Attenuation $f_{i} f_{j}^{\star}$ resulting from various filtering sources as discussed in Appendix B: (a) parasitic filtering of the position sensing devices (Eq. (B1)) using the parameters from Table I for the $\perp$ direction, (b) different electric low-pass filters (Eqs. (B3) and (B4)), (c) uniform averaging of signals over a time window of duration $\tau_{\text {av }}$ (Eq. (B5)), (d) time delay between the two recorded signals (Eq. (B7)). Positive values of $f_{i} f_{j}^{\star}$ are drawn as solid lines, negative ones are dashed.

Auto-PSDs thus remain unaffected by time shifts, $\left|f_{i}(\omega)\right|^{2}$ $=1$, while the cross-PSD are multiplied by a complex factor

$$
f_{i}^{\mathrm{del}}(\omega) f_{j}^{\mathrm{del}^{\star}}(\omega)=\cos \omega \Delta \tau_{\mathrm{del} i, j}+\mathrm{i} \sin \omega \Delta \tau_{\mathrm{del} i, j},
$$

with relative time delay between the channels

$$
\Delta \tau_{\mathrm{del} i, j} \equiv \tau_{\mathrm{del}, i}-\tau_{\mathrm{del}, j} .
$$

For $\Delta \tau_{\mathrm{del} i, j} \neq 0$, cross-PSDs are modified as shown in Fig. 10(d). 


\section{Overall transfer functions}

Given the specifications of our signal recording hardware (Sec. II), time averaging effects as well as temporal delays in the signal detection are negligible. The overall filter function for channel $i$ is therefore given by

$$
f_{i}(\omega)=f_{i}^{\mathrm{pf}}(\omega) f_{i}^{\mathrm{B} 8}(\omega),
$$

accounting for the parasitic filtering of the position sensing device (Appendix B 1) and for the Butterworth filter of the amplification device (Appendix B 2); according to Eq. (B1), the above expression involves two parameters $\lambda_{i}$ and $\tau_{i}$, for which explicit values are determined in the instrumental calibration in Sec. VII.

\section{APPENDIX C: PERIODOGRAM COVARIANCES}

We explicitly derive the periodogram covariances for an arbitrary number of signals $z_{i}(t)$ recorded over a time window $T$, again neglecting leakage effects (cf. Appendix D 1). According to Secs. III B and V, the Fourier components of the signals

$$
z_{i}\left(\omega_{n}\right)=\sum_{j} A_{i j}\left(\omega_{n}\right) v_{j}\left(\omega_{n}\right),
$$

linearly depend on the stochastic Fourier components $v_{j}$ representing thermal forces and instrumental noise, where the $A_{i j}$ denote complex coefficients resulting from the underlying equations of motion and the signal processing in the instrument. For a stationary stochastic process, the real and imaginary parts of the stochastic Fourier components are assumed to be normal distributed random variables with vanishing mean and with (cross-) correlations

$$
\begin{aligned}
\left\langle\operatorname{Re}\left[v_{i}\left(\omega_{n}\right)\right] \operatorname{Re}\left[v_{j}\left(\omega_{n^{\prime}}\right)\right]\right\rangle & =\left\langle\operatorname{Im}\left[v_{i}\left(\omega_{n}\right)\right] \operatorname{Im}\left[v_{j}\left(\omega_{n^{\prime}}\right)\right]\right\rangle \\
& =T \sigma_{i j}^{2}\left(\omega_{n}\right) \delta_{n n^{\prime}},
\end{aligned}
$$

where $\sigma_{i j}^{2}$ denotes the corresponding variance and $\delta_{n n^{\prime}}$ the Kronecker symbol, meaning that Fourier components corresponding to distinct frequencies are uncorrelated. Real and imaginary parts of the $v_{i}$ components are independent due to time reversal symmetry and thus

$$
\left\langle\operatorname{Re}\left[v_{i}\left(\omega_{n}\right)\right] \operatorname{Im}\left[v_{j}\left(\omega_{n^{\prime}}\right)\right]\right\rangle=0 .
$$

Note that Eq. (C1) is quite general in the sense that it describes the electric signals in any experimental setup, where the thermal motion of the compounds is described by linear integro-differential equations of motion (e.g., Eq. (8)), the signal processing is linear (Eq. (17)), and instrumental noise contributing to the signal is additive (Eq. (18)); the number of stochastic components $v_{i}$ is not restricted. As a result, the periodograms

$$
P_{i j}\left(\omega_{n}\right) \equiv \frac{z_{i}\left(\omega_{n}\right) z_{j}^{\star}\left(\omega_{n}\right)}{T}=\frac{1}{T} \sum_{k, l} A_{i k} A_{j l}^{\star} v_{k}\left(\omega_{n}\right) v_{l}^{\star}\left(\omega_{n}\right),
$$

are again (complex) random variables, the statistics of which can be inferred from the properties of the $v_{i}$ components given above.
In the following, the frequency dependence is implicitly assumed, though the argument $\omega_{n}$ is omitted to keep the notation simple. Basic algebra and Gaussian statistics yield

$$
\begin{gathered}
\left\langle v_{i} v_{j}^{\star}\right\rangle=2 T \sigma_{i j}^{2}, \\
\left\langle v_{i} v_{j}^{\star} v_{k} v_{l}^{\star}\right\rangle=4 T^{2}\left(\sigma_{i j}^{2} \sigma_{k l}^{2}+\sigma_{i l}^{2} \sigma_{j k}^{2}\right) .
\end{gathered}
$$

Real and imaginary parts of a periodogram are given by

$$
\begin{aligned}
& \operatorname{Re}\left[P_{i j}\right]=\frac{1}{2} \frac{1}{T} \sum_{k, l}\left(A_{i k} A_{j l}^{\star} v_{k} v_{l}^{\star}+A_{i k}^{\star} A_{j l} v_{k}^{\star} v_{l}\right), \\
& \operatorname{Im}\left[P_{i j}\right]=\frac{1}{2 \mathrm{i}} \frac{1}{T} \sum_{k, l}\left(A_{i k} A_{j l}^{\star} v_{k} v_{l}^{\star}-A_{i k}^{\star} A_{j l} v_{k}^{\star} v_{l}\right) .
\end{aligned}
$$

In the limit $T \rightarrow \infty$, their expectation values by definition (cf. Eqs. (21) and (25)) correspond to the real and imaginary parts of the corresponding PSDs

$$
\begin{aligned}
& \left\langle\operatorname{Re}\left[P_{i j}\right]\right\rangle=2 \sum_{k, l} \operatorname{Re}\left[A_{i k} A_{j l}^{\star}\right] \sigma_{k l}^{2}=\operatorname{Re}\left[S_{i j}\right], \\
& \left\langle\operatorname{Im}\left[P_{i j}\right]\right\rangle=2 \sum_{k, l} \operatorname{Im}\left[A_{i k} A_{j l}^{\star}\right] \sigma_{k l}^{2}=\operatorname{Im}\left[S_{i j}\right] .
\end{aligned}
$$

Similarly, making use of the relations in Eq. (C4), higher moments of real and imaginary parts of the periodograms can be calculated. Gaussian statistics allow to reformulate the resulting expressions in terms of real and imaginary parts of the PSDs yielding covariances

$$
\begin{aligned}
& \left\langle\operatorname{Re}\left[P_{i j}\right] \operatorname{Re}\left[P_{k l}\right]\right\rangle-\left\langle\operatorname{Re}\left[P_{i j}\right]\right\rangle\left\langle\operatorname{Re}\left[P_{k l}\right]\right\rangle \\
& =\frac{1}{2}\left(\operatorname{Re}\left[S_{i k}\right] \operatorname{Re}\left[S_{j l}\right]+\operatorname{Im}\left[S_{i k}\right] \operatorname{Im}\left[S_{j l}\right]\right. \\
& \left.+\operatorname{Re}\left[S_{i l}\right] \operatorname{Re}\left[S_{j k}\right]+\operatorname{Im}\left[S_{i l}\right] \operatorname{Im}\left[S_{j k}\right]\right), \\
& \left\langle\operatorname{Re}\left[P_{i j}\right] \operatorname{Im}\left[P_{k l}\right]\right\rangle-\left\langle\operatorname{Re}\left[P_{i j}\right]\right\rangle\left\langle\operatorname{Im}\left[P_{k l}\right]\right\rangle \\
& =\frac{1}{2}\left(\operatorname{Re}\left[S_{i k}\right] \operatorname{Im}\left[S_{j l}\right]-\operatorname{Im}\left[S_{i k}\right] \operatorname{Re}\left[S_{j l}\right]\right. \\
& \left.+\operatorname{Im}\left[S_{i l}\right] \operatorname{Re}\left[S_{j k}\right]-\operatorname{Re}\left[S_{i l}\right] \operatorname{Im}\left[S_{j k}\right]\right), \\
& \left\langle\operatorname{Im}\left[P_{i j}\right] \operatorname{Im}\left[P_{k l}\right]\right\rangle-\left\langle\operatorname{Im}\left[P_{i j}\right]\right\rangle\left\langle\operatorname{Im}\left[P_{k l}\right]\right\rangle \\
& =\frac{1}{2}\left(\operatorname{Re}\left[S_{i k}\right] \operatorname{Re}\left[S_{j l}\right]+\operatorname{Im}\left[S_{i k}\right] \operatorname{Im}\left[S_{j l}\right]\right. \\
& \left.-\operatorname{Re}\left[S_{i l}\right] \operatorname{Re}\left[S_{j k}\right]-\operatorname{Im}\left[S_{i l}\right] \operatorname{Im}\left[S_{j k}\right]\right) .
\end{aligned}
$$

Again, these approximations are strictly exact only in the limit $T \rightarrow \infty$. For the case of two detected signals, the variances $(k=i, l=j$ ) resulting from the above expressions are given and discussed in Sec. VI A; the overall covariance matrix for the averaged periodograms of two signals is found in Sec. VI B.

\section{APPENDIX D: DISCRETE FOURIER TRANSFORMS}

Consider a continuous signal $z(t)$ of infinite duration, which is sampled with finite sampling rate $f_{\text {sampl }} \equiv 1 / \Delta t$ over a time window of duration $T \equiv N \Delta t$. Applying a discrete 
Fourier transform (DFT) to $z(t)$ yields $N$ discrete Fourier components

$$
Z(n) \equiv \sum_{m=0}^{N-1} z(m \Delta t) \mathrm{e}^{\mathrm{i} 2 \pi m n / N} \approx \frac{z\left(\omega_{n}\right)}{\Delta t},
$$

where $z\left(\omega_{n}\right)$ denotes the value of the Fourier transformation of the original, continuous signal of infinite duration at the angular frequency $\omega_{n} \equiv 2 \pi n /(N \Delta t)$ with $n$ being integer. Due to the finite time resolution and the overall duration of the signal, the correspondence between discrete and continuous Fourier components in Eq. (D1) is only approximate; in the following, we review the relationship between discrete and continuous Fourier transformations and point out which precautions have to be taken when working with DFTs. ${ }^{40}$

Equation (D1) can be reformulated as

$$
Z(n)=\int_{-\infty}^{\infty} \mathrm{d} t \operatorname{Pulse}_{T}(t) \operatorname{Comb}_{\Delta t}(t) z(t) \mathrm{e}^{\mathrm{i} \omega_{n} t},
$$

where we defined the functions

$$
\begin{gathered}
\text { Pulse }_{T}(t) \equiv \Theta(t+\Delta t / 2) \Theta(-t+T-\Delta t / 2), \\
\operatorname{Comb}_{\Delta t}(t) \equiv \sum_{m=-\infty}^{\infty} \delta(t-m \Delta t) .
\end{gathered}
$$

The discrete Fourier components $Z$ thus correspond to the continuous Fourier transform of the product of the rectangular pulse function Pulse ${ }_{T}$, the Dirac comb function $\mathrm{Comb}_{\Delta t}$ and the continuous and infinite signal $z$. According to the convolution theorem (Eq. (A3))

$$
Z(n)=(2 \pi)^{-2} \operatorname{Pulse}_{T} * \operatorname{Comb}_{\Delta t} * z\left(\omega_{n}\right),
$$

involving two convolutions: the first one arises from the finite window length $T$ and is responsible for spectral leakage of the DFT, the second one is due to the finite sampling time interval $\Delta t$ and causes aliasing; both effects are discussed in the following.

\section{Spectral leakage}

The finite window time $T$ causes a convolution with

$$
\text { Pulse }_{T}(\omega)=2 \frac{\sin (\omega T / 2)}{\omega} \mathrm{e}^{\mathrm{i} \omega(T-\Delta t) / 2},
$$

a highly oscillating function with side-lobes decaying as $1 / \omega$. Fourier components around $\omega_{n}$ (mainly in a region of width $2 \pi / T)$ thus contribute to $Z(n)$ defined in Eq. (D1). The effect of spectral leakage can be reduced by using window functions, ${ }^{41}$ which smoothly fade in and out the signal and thereby reduce the side lobe amplitudes in frequency space (at the cost of a wider central peak). Spectral leakage causes prominent artifacts in the DFTs of periodic signals in case of a mismatch of window time and period of the signal; since thermal PSDs however smoothly depend on frequency, leakage effects are of minor importance and can be further minimized by increasing the window length $T$.

\section{Aliasing}

The finite sampling interval $\Delta t$ causes the convolution of the continuous Fourier transform with

$$
\operatorname{Comb}_{\Delta t}(\omega)=\frac{2 \pi}{\Delta t} \sum_{m=-\infty}^{\infty} \delta\left(\omega+m \frac{2 \pi}{\Delta t}\right)
$$

According to the sampling theorem, Fourier components with frequencies $|\omega|>2 \pi f_{\mathrm{Nyq}} \equiv \pi / \Delta t$ are mapped onto Fourier components within the Nyquist interval. In fact, in the limit of large sample sizes $(N \rightarrow \infty)$ Eq. (D5) reduces to

$$
Z(n)=\frac{1}{\Delta t} \sum_{m=-\infty}^{\infty} z\left(\omega_{n}+m \frac{2 \pi}{\Delta t}\right) \equiv \frac{1}{\Delta t} z^{\text {al }} \cdot\left(\omega_{n}\right),
$$

the aliased version of the continuous Fourier transform. For functions which quickly decay beyond the Nyquist interval, the effect of aliasing is most prominent at its edges $(|\omega|$ $\lesssim 2 \pi f_{\mathrm{Nyq}}$ ); aliasing effects and the (mis-)use of anti-aliasing filters are discussed in detail in Ref. 9.

\section{Calculating periodograms via DFT}

The cross-PSD of two signals $z_{i}$ and $z_{j}$ is estimated by calculating the periodogram

$$
P_{i j}(n) \equiv \frac{\Delta t}{N} Z_{i}(n) Z_{j}^{\star}(n),
$$

where $Z_{i}$ and $Z_{j}$ denote the DFTs of $z_{i}$ and $z_{j}$; in practice, DFTs of time series are efficiently computed using fast Fourier transform algorithms. According to Eq. (D5) the expectation value is

$$
\left\langle P_{i j}(n)\right\rangle=\frac{\Delta t}{2 \pi} \int_{-\infty}^{\infty} \mathrm{d} \omega S_{i j}\left(\omega_{n}-\omega\right) \frac{1}{N}\left(\frac{\sin (N \omega \Delta t / 2)}{\sin (\omega \Delta t / 2)}\right)^{2},
$$

which, using Fejer's theorem, in the limit of large sample sizes $N$, reduces to the aliased PSD defined in Eq. (23)

$$
\lim _{N \rightarrow \infty}\left\langle P_{i j}(n)\right\rangle=\sum_{m=-\infty}^{\infty} S_{i j}\left(\omega_{n}+m \frac{2 \pi}{\Delta t}\right) .
$$

The double-infinite sum is in our case approximated numerically using upper and lower cutoffs $m_{\max }=-m_{\min }=5$, where cutoff errors are negligible due to the attenuation characteristics of the employed eighth order Butterworth filter (cf. Sec. II and Appendix B 2).

\section{APPENDIX E: NORMAL DISTRIBUTED VARIABLES ON LOGARITHMIC SCALES}

As discussed in Sec. VI, the values of averaged periodograms $\bar{P}_{i j}$ are normal distributed random variables. The frequency dependence of periodograms and PSDs is generally displayed on logarithmic graphs; a short calculation demonstrates what has to be kept in mind, when interpreting such graphs.

The PDF for a normal distributed variable $x$ with mean $\mu$ and variance $\sigma^{2}$ is given by

$$
\mathcal{P}(x)=\frac{1}{\sqrt{2 \pi \sigma^{2}}} \exp \left(-\frac{(x-\mu)^{2}}{2 \sigma^{2}}\right),
$$


and thus random variables $x$ scatter around $\mu$, if a linear axis is used. However, in a logarithmic plot, the variables shown are (taking into account the variable's sign)

$$
y_{ \pm} \equiv \log ( \pm x) \Leftrightarrow x= \pm \exp \left(y_{ \pm}\right) .
$$

This coordinate change affects the form of the PDF, which have to fulfill

$$
\tilde{\mathcal{P}}_{ \pm}\left(y_{ \pm}\right)\left|\mathrm{d} y_{ \pm}\right|=\mathcal{P}(x) \mathrm{d} x .
$$

The PDFs for the variables $y_{ \pm}$therefore read

$$
\begin{aligned}
\tilde{\mathcal{P}}_{ \pm}\left(y_{ \pm}\right) & =\mathcal{P}(x)\left|\frac{\mathrm{d} x}{\mathrm{~d} y_{ \pm}}\right| \\
& =\frac{1}{\sqrt{2 \pi \sigma^{2}}} \exp \left(y_{ \pm}-\frac{\left( \pm \exp \left(y_{ \pm}\right)-\mu\right)^{2}}{2 \sigma^{2}}\right) .
\end{aligned}
$$

Note that $\mathcal{P}$ is peaked around $x^{*}=\mu$, while the PDFs $\tilde{\mathcal{P}}_{ \pm}$are peaked around

$$
y_{ \pm}^{*}=\log \left(\frac{ \pm \mu+\sqrt{\mu^{2}+4 \sigma^{2}}}{2}\right),
$$

with limiting cases (assuming $\mu$ positive)

$$
\lim _{\sigma \rightarrow 0} \exp \left(y_{+}^{*}\right)=\mu, \quad \lim _{\mu \rightarrow 0} \exp \left(y_{ \pm}^{*}\right)=\sigma .
$$

As long as the statistical uncertainty is much smaller than the magnitude of the expectation value, random variables accumulate as expected around $\mu$ on a logarithmic plot. In the opposite case, random variables tend to scatter (with varying sign) around $\sigma$. Depending on the ratio of expectation value and statistical uncertainty, the absolute values of averaged cross-periodograms may therefore reflect the frequency dependence of the cross-PSD or its statistical uncertainty only as discussed in Sec. VIII B.

${ }^{1}$ J. R. Moffitt, Y. R. Chemla, S. B. Smith, and C. Bustamante, Annu. Rev. Biochem. 77, 205 (2008).

${ }^{2}$ H. Dietz, F. Berkemeier, M. Bertz, and M. Rief, Proc. Natl. Acad. Sci. U.S.A. 103, 12724 (2006); M. T. Woodside, P. C. Anthony, W. M. BehnkeParks, K. Larizadeh, D. Herschlag, and S. M. Block, Science 314, 1001 (2006).

${ }^{3}$ J. C. M. Gebhardt, T. Bornschlögl, and M. Rief, Proc. Natl. Acad. Sci. U.S.A. 107, 2013 (2010); J. Stigler, F. Ziegler, A. Gieseke, J. C. M. Gebhardt, and M. Rief, Science 334, 512 (2011)

${ }^{4}$ T. Franosch, M. Grimm, M. Belushkin, F. M. Mor, G. Foffi, L. Forro, and S. Jeney, Nature (London) 478, 85 (2011); A. Jannasch, M. Mahamdeh, and E. Schaffer, Phys. Rev. Lett. 107, 228301 (2011).

${ }^{5}$ F. Gittes, B. Schnurr, P. D. Olmsted, F. C. MacKintosh, and C. F. Schmidt, Phys. Rev. Lett. 79, 3286 (1997); J. C. Crocker, M. T. Valentine, E. R. Weeks, T. Gisler, P. D. Kaplan, A. G. Yodh, and D. A. Weitz, ibid. 85, 888 (2000); L. Starrs and P. Bartlett, Faraday Discuss. 123, 323 (2003).

${ }^{6}$ J. R. Moffitt, Y. R. Chemla, D. Izhaky, and C. Bustamante, Proc. Natl. Acad. Sci. U.S.A. 103, 9006 (2006).

${ }^{7}$ M. Hinczewski, Y. von Hansen, and R. R. Netz, Proc. Natl. Acad. Sci. U.S.A. 107, 21493 (2010).
${ }^{8}$ P. P. Bloomfield, Fourier Analysis of Time Series: An Introduction (Wiley, 1976).

${ }^{9}$ K. Berg-Sorensen and H. Flyvbjerg, Rev. Sci. Instrum. 75, 594 (2004).

${ }^{10}$ I. M. Tolic-Norrelykke, K. Berg-Sorensen, and H. Flyvbjerg, Comput. Phys. Commun. 159, 225 (2004).

${ }^{11}$ K. Berg-Sorensen, E. J. G. Peterman, T. Weber, C. F. Schmidt, and H. Flyvbjerg, Rev. Sci. Instrum. 77, 063106 (2006).

${ }^{12}$ S. F. Tolic-Norrelykke, E. Schäffer, J. Howard, F. S. Pavone, F. Julicher, and H. Flyvbjerg, Rev. Sci. Instrum. 77, 103101 (2006).

${ }^{13}$ S. F. Norrelykke and H. Flyvbjerg, Rev. Sci. Instrum. 81, 075103 (2010).

${ }^{14}$ M. Atakhorrami, G. H. Koenderink, C. F. Schmidt, and F. C. MacKintosh, Phys. Rev. Lett. 95, 208302 (2005); T. B. Liverpool and F. C. MacKintosh, ibid. 95, 208303 (2005); M. Atakhorrami, D. Mizuno, G. H. Koenderink, T. B. Liverpool, F. C. MacKintosh, and C. F. Schmidt, Phys. Rev. E 77, 061508 (2008).

${ }^{15}$ E. Fällman and O. Axner, Appl. Opt. 36, 2107 (1997).

${ }^{16}$ P. Mangeol and U. Bockelmann, Rev. Sci. Instrum. 79, 083103 (2008).

${ }^{17}$ M. Atakhorrami, K. M. Addas, and C. F. Schmidt, Rev. Sci. Instrum. 79, 043103 (2008).

${ }^{18}$ X. Perpina, X. Jorda, M. Vellvehi, J. Millan, and N. Mestres, Rev. Sci. Instrum. 76, 025106 (2005).

${ }^{19}$ L. Landau and E. Lifshitz, Statistical Physics, Part 1, 3rd ed., Course of Theoretical Physics Vol. 5 (Butterworth-Heinemann, Oxford, UK, 1980).

${ }^{20}$ J. C. Meiners and S. R. Quake, Phys. Rev. Lett. 82, 2211 (1999); P. Bartlett, S. I. Henderson, and S. J. Mitchell, Philos. Trans. R. Soc. London, Ser. A 359, 883 (2001); S. Henderson, S. Mitchell, and P. Bartlett, Phys. Rev. E 64, 061403 (2001).

${ }^{21}$ J. C. Meiners and S. R. Quake, Phys. Rev. Lett. 84, 5014 (2000).

${ }^{22}$ C. Bustamante, Y. R. Chemla, and J. R. Moffit, "High-resolution dual-trap optical tweezers with differential detection," Single-Molecule Techniques: A Laboratory Manual, edited by P. R. Selvin and T. Ha (Cold Spring Harbor Laboratory, New York, USA, 2008).

${ }^{23}$ K. C. Neuman and S. M. Block, Rev. Sci. Instrum. 75, 2787 (2004).

${ }^{24}$ G. Stokes, Proc. Cambridge Philos. Soc. 9, 8 (1851).

${ }^{25}$ D. A. Weitz, D. J. Pine, P. N. Pusey, and R. J. A. Tough, Phys. Rev. Lett. 63, 1747 (1989); H. J. H. Clercx and P. P. J. M. Schram, Phys. Rev. A 46, 1942 (1992).

${ }^{26}$ A. Erbas, R. Podgornik, and R. R. Netz, Eur. Phys. J. E 32, 147 (2010).

${ }^{27} \mathrm{See}$ http://www.physik.fu-berlin.de/en/einrichtungen/ag/ag-netz/movies/ hydrodynamic_flow for the series of flow field animations.

${ }^{28}$ W. van Saarloos and P. Mazur, Physica A 120, 77 (1983).

${ }^{29}$ J. Rotne and S. Prager, J. Chem. Phys. 50, 4831 (1969).

${ }^{30}$ D. L. Ermak and J. A. McCammon, J. Chem. Phys. 69, 1352 (1978).

${ }^{31} \mathrm{C}$. W. Oseen, Neuere Methoden und Ergebnisse in der Hydrodynamik (Akademische Verlagsgesellschaft, 1927).

${ }^{32}$ S. Kim and S. J. Karrila, Microhydrodynamics - Principles and Selected Applications (Dover, Mineola, 1991).

${ }^{33}$ B. H. Zimm, J. Chem. Phys. 24, 269 (1956)

${ }^{34}$ M. Reichert and H. Stark, Phys. Rev. E 69, 031407 (2004); S. Martin, M. Reichert, H. Stark, and T. Gisler, Phys. Rev. Lett. 97, 248301 (2006).

${ }^{35}$ E. J. G. Peterman, M. A. van Dijk, L. C. Kapitein, and C. F. Schmidt, Rev. Sci. Instrum. 74, 3246 (2003).

${ }^{36}$ J. D. Scargle, Astrophys. J. 263, 835 (1982); J. H. Horne and S. L. Baliunas, ibid. 302, 757 (1986).

${ }^{37}$ M. Lourakis, "levmar: Levenberg-marquardt nonlinear least squares algorithms in c/c++," see http://www.ics.forth.gr/ lourakis/levmar/.

${ }^{38}$ See the Supplementary material at http://dx.doi.org/10.1063/1.4753917 for the explanation of the observed crosstalk asymmetry.

${ }^{39}$ K. Berg-Sorensen, L. Oddershede, E. L. Florin, and H. Flyvbjerg, J. Appl. Phys. 93, 3167 (2003).

${ }^{40}$ H. J. Weaver, Theory of Discrete and Continuous Fourier Analysis (Wiley, 1989).

${ }^{41}$ W. Press, S. Teukolsky, W. Vetterling, and B. Flannery, Numerical Recipes in $C$, 2nd ed. (Cambridge University Press, Cambridge, UK, 1992). 